\title{
SYNERGIC RENOPROTECTIVE EFFECTS OF COMBINED ASC THERAPY WITH RAAS BLOCKADE IN EXPERIMENTAL ADVANCED CKD
}

Marina PC Maires ${ }^{1}$, Krislley R Pereira ${ }^{1}$, Everidiene KVB Silva ${ }^{1}$, Victor HR Souza ${ }^{1}$, Flavio Teles$^{2}$, Paulyana F Barbosa², Margoth R Garnica ${ }^{1}$, Felipe M Ornellas ${ }^{1}$, Irene L Noronha ${ }^{1}$, Camilla Fanelli ${ }^{1 *}$

${ }^{1}$ Hospital das Clínicas da Faculdade de Medicina da Universidade de São Paulo, São Paulo, Brazil

${ }^{2}$ Universidade Estadual de Ciências da Saúde de Alagoas, Maceió, Brazil

\section{Correspondence address}

Camilla Fanelli, PhD

Laboratório de Nefrologia Celular, Genética e Molecular

Faculdade de Medicina - Universidade de São Paulo

Av. Dr. Arnaldo, 455, 40 andar, 4304

São Paulo, CEP: 01246-903, Brasil

Tel: +5511984320508

Email: camilla.fanelli@usp.br 


\section{ABSTRACT}

Global prevalence of chronic kidney disease (CKD) has increased considerably in the recent decades. Overactivity of the renin-angiotensin-aldosterone system (RAAS), associated to renal inflammation and fibrosis contribute to its evolution. The treatments currently employed to control CKD progression are limited and mainly based on the pharmacological inhibition of RAAS, associated with diuretics and immunosuppressive drugs. However, this conservative management promotes only partial deceleration of CKD evolution, and does not completely avoid the progression of the disease and the loss of renal function, which motivates the medical and scientific community to investigate new therapeutic approaches to detain renal inflammation / fibrosis and CKD progression. Recent studies have shown the application of mesenchymal stem cells (mSC) to exert beneficial effects on the renal tissue of animals submitted to experimental models of CKD. In this context, the aim of the present study was to evaluate the effects of subcapsular application of adipose tissue-derived $\mathrm{mSC}$ (ASC) in rats submitted to the $5 / 6$ renal ablation model, 15 days after the establishment of CKD, when the nephropathy was already severe. We also verify whether ASC associated to Losartan, would promote greater renoprotection when compared to the respective monotherapies. Animals were followed until 30 days of CKD, when body weight, systolic blood pressure, biochemical, histological, immunohistochemical and gene expression analysis were performed. The combination of ASC and Losartan was more effective than Losartan monotherapy in reducing systolic blood pressure and glomerulosclerosis, and also promoted the complete normalization of proteinuria and albuminuria, a significant reduction in renal interstitial macrophage infiltration and downregulation of renal IL-6 gene expression. The beneficial effects of ACS are possibly due to the immunomodulatory and antiinflammatory role of factors secreted by these cells, modulating the local immune response. Although studies are still required, our results demonstrated that a subcapsular inoculation of ASC, associated with the administration of Losartan, exerted additional renoprotective effect in rats submitted to a severe model of established CKD, when compared to Losartan monotherapy, thus suggesting ASC may be a potential adjuvant to RAAS-blockade therapy currently employed in the conservative management of CKD.

Key words: Mesenchymal stem cells, Chronic kidney disease, Cell therapy 


\section{INTRODUCTION}

The prevalence of chronic kidney disease (CKD) has been increasing worldwide in recent decades $[1,2]$. It is estimated that CKD affects around $10 \%$ of the global population, leading to progressive loss of renal function and the need for renal replacement therapy (RRT) [3]. Regardless of its etiology, CKD usually progresses with hemodynamic changes, such as increased glomerular pressure and the emergence of systemic hypertension, loss of the kidney filtration barrier selectivity, characterized by proteinuria and albuminuria, serum urea nitrogen and creatinine retention, and the establishment of local renal inflammation, with leukocyte infiltration, increased extracellular matrix (ECM) deposition, development of renal fibrosis and progressive renal failure [4,5].

Some of the pathophysiological mechanisms known to contribute to CKD progression include the overactivity of the renin-angiotensin-aldosterone system (RAAS), with consequent increase in the biological effects of Angiotensin II (AlI), and the activation of local innate and adaptive immune responses which trigger the production of proinflammatory mediators, leukocyte recruitment and ECM synthesis [4-9]. Therefore, pharmacological suppression of RAAS with All receptor blockers (ARBs), such as Losartan, and/or All converting enzyme (ACE) inhibitors (ACEIs) are the most employed therapeutic strategy in the conservative treatment of CKD. However, although widely applied, RAAS inhibitors alone are not able to revert kidney damage, nor to completely detain CKD progression. Until the present moment there is no specific drug or therapy to treat CKD and to effectively to stop the progression of renal inflammation and fibrosis at once, which motivates the scientific community to investigate experimental and pre-clinical strategies to detain or retard CKD progression [2]. 
In this context, recent studies have shown promising results with the therapeutic application of mesenchymal stromal cells (mSC) in reducing renal inflammation and fibrosis, in experimental models of CKD [4, 10-12]. According to the current literature, the administration of mSC would not exert its beneficial effects due to direct cell differentiation and tissue replacement, which was previously believed to be the main biological mechanism responsible for the renoprotection achieved with cell therapy, but through paracrine immunomodulatory effects, which may involve the synthesis and secretion of anti-inflammatory cytokines and interleukins [13,14]. Cavaglieri and collaborators demonstrated that bone marrow-derived mSC (BMSC) protected Wistar rats submitted to the 5/6 renal ablation model of CKD from the development of hypertension, albuminuria and glomerulosclerosis, when administered in the renal subcapsular space, concomitantly with the surgical induction of nephropathy, as a preventive strategy. The authors showed the inoculation via renal subcapsular route to provide effective cell migration through the organ cortex, glomeruli and renal interstitium, presenting satisfactory cell distribution and permanence in the region of interest, throughout the study period [10].

Corroborating these findings, Ornellas and co-authors shown mSC derived from the adipose tissue (ASC) to be effective in preventing renal inflammation, proteinuria and podocyte injury when given to animals submitted to the puromycin aminonucleosideinduced nephrosis model [12]. Xing and collaborators demonstrated that the administration of BMSC through the tail vein of animals submitted to a unilateral ureteral obstruction model exerted a renoprotective effect, attenuating interstitial fibrosis and inhibiting the loss of peritubular capillaries in this model [15]. In line with these findings, Pepineli and co-authors more recently demonstrated that ASC modulates the local 
inflammatory response in a rat model of chronic allograft nephropathy, induced by the allogeneic kidney transplantation using Fisher 344 rats as donors and Lewis rats as graft recipients. In this elegant model, ASC treatment reduced renal macrophage infiltration, as well as the local expression of proinflammatory and pro-fibrotic factors, such as IL-1B, IL-6, and TNF- $\alpha[16]$.

As mentioned above, there are currently a number of research articles demonstrating positive effects of ASC cell therapy in preventing the development of CKD, in different animal models. However, in none of these studies did the authors verify whether the late renal $\mathrm{mSC}$ inoculation would also be effective in halting the progression of the already established nephropathy, or even in reversing kidney damage. Thus, the aim of the present study was to investigate the renoprotective effects of a late single renal subcapsular application of ASC, administered 15 days after the induction of CKD, when all clinical and laboratorial characteristics of renal failure where already evident, in rats submitted to the 5/6 renal ablation model, in order to more closely resemble the clinical settings observed in humans. In addition, we sought to verify whether ASC inoculation, associated to the oral administration of Losartan, would promote greater renoprotection compared to ARB monotherapy, in this same CKD model, in order to analyze whether ASC therapy could be considered as adjuvant to the currently employed pharmacological treatment to control CKD progression. 


\section{MATERIAL AND METHODS}

\section{Animal Model}

Ninety male Wistar rats, weighing 220-280 g, were obtained from the local animal facility of the University of São Paulo (USP). Animals were kept at a constant temperature of 23 $\pm 1^{\circ} \mathrm{C}, 5 \%$ relative air humidity, under $12 \mathrm{~h}$ artificial light/dark cycle, and had free access to rodent chow and tap water throughout the period of analysis. The experimental protocol followed in the present study was fully approved by the Ethics Committee on the Use of Experimental Animals of the University of São Paulo Medical School (CEUAFMUSP No 1019/2018).

Seventy of the above-mentioned rats were submitted to the $5 / 6$ renal ablation model of CKD, through a one-step surgical procedure: Animals were anesthetized with inhalation anesthesia with isoflurane (Bio Chimico, Brazil), and underwent a ventral laparotomy, under aseptic conditions. The infarction of two-thirds of the left kidney was obtained by the ligation of two of the three branches of the left renal artery, followed by total nephrectomy of the right kidney. Additional 11 rats, used as controls, were also submitted to isoflurane anesthesia and ventral laparotomy, but with no removal of renal mass (Sham). After surgery, all animals were kept in heated cages until they recovered from anesthesia. Post-operative care included a single dose of antibiotic (IM injection of $0.4 \mathrm{~mL} / \mathrm{kg}$ Enrofloxacin 5\%, Bayer), and 3 doses of analgesic (SC injections of $5 \mathrm{mg} / \mathrm{kg}$ Tramadol), one every 24 hours. 


\section{Isolation, Maintenance, Characterization and Inoculation of ASC}

Adipose-derived mesenchymal stromal cells (ASC) were isolated from the perigonadal adipose tissue of 3 healthy adult male Wistar rats. The animals were anesthetized by isoflurane inhalation and had their blood directly collected from the abdominal aorta to later obtain normal rat serum (NRS), employed in the in vitro experiments. After blood collection, the animals had the perigonadal adipose tissue removed, minced and digested with $0.075 \%$ collagenase solution (Sigma-Aldrich, USA). After centrifugation and processing, the cell pellet was resuspended in $10 \mathrm{~mL}$ of complete DMEM-Low medium $(10 \%$ FBS $)$ and plated in cell culture flasks, which were kept in a humid oven at $37^{\circ} \mathrm{C}, 5 \%$ $\mathrm{CO}_{2}$ (Thermo Fisher Scientific, Marietta, USA). The cells were maintained in culture, being monitored daily under inverted microscopy. Culture medium changes were performed three times a week and cells were trypsinized and replated whenever they reached a confluence between $60 \%$ and $80 \%$.

Between the $4^{\text {th }}$ and $6^{\text {th }}$ cell passages, cell viability analysis was performed, using trypan blue staining, followed by the characterization of cell populations through flow cytometry FacsCanto (Becton Dickinson, San Jose, EUA). For this characterization, the presence of specific cellular markers for mSC, such as CD29, CD44, CD90 and CD105, as well as the absence of the pan-leukocyte marker CD45 were verified, using specific monoclonal antibodies [17]. The results were analyzed in the form of histograms of the cell population with positive reaction for each antibody, thus characterizing the $\mathrm{mSC}$ population, as shown in Supplementary Figure 1. Further cell plasticity tests were performed to verify the ability of ASC to differentiate in osteogenic, chondrogenic and adipogenic cell lines, using a commercially available kit (STEMPRO ${ }^{\circledR}$ Osteocyte / Condrocyte / Adipocyte 
Differentiation), also shown in Supplementary Figure 1. After characterization, ASC were collected from culture flasks and divided into samples containing $2 \times 10^{6}$ cells resuspended in $10 \mu \mathrm{L}$ of sterile PBS (cell inoculums). The animals were anesthetized with isoflurane, underwent ventral laparotomy and were submitted to the subcapsular injection of the cell inoculum, followed by the same post-operative care mentioned above.

\section{Experimental Protocol}

The animals had their body weight (BW, g) monitored weekly and their systolic blood pressure (SBP, mmHg) measured with an automated optoelectronic device (Visitech Systems, Apex, NC) every 15 days, when 24-hour urine samples were also collected to verify changes in urinary volume (UV, $\mathrm{mL}$ ), urinary protein excretion (UPE, $\mathrm{mg} / 24 \mathrm{~h})$ by colorimetric analysis (Kit Sensiprot, Labtest \# 36, Brazil) and urinary albumin excretion ( $U A E, m g / 24 h$ ), by radial immunodiffusion, using a specific anti-rat albumin antibody (MPBiomedicals LLC \#55711, USA) [18].

On the $15^{\text {th }}$ day after renal ablation, rats were distributed into five experimental groups, randomized according to their basal UPE, as follows: basal CKD ( $N=11)$, euthanized 15 days after CKD induction; CKD (N=13), kept untreated until the $30^{\text {th }}$ day after $5 / 6$ renal ablation; CKD+ASC ( $N=15)$, that received a subcapsular injection of ASC after 15 days of renal ablation and were followed until the $30^{\text {th }}$ day after CKD induction; CKD+LOS $(N=12)$, that received $50 \mathrm{mg} / \mathrm{Kg} /$ day of Losartan, diluted in drinking water, from the $15^{\text {th }}$ to the $30^{\text {th }}$ days after CKD induction; and CKD+ASC+LOS $(N=14)$, that received both the ASC subcapsular injection and the oral treatment with Losartan, as illustrated in Supplementary Figure 2. Additionally, 3 animals submitted to CKD induction were euthanized to collect total blood and obtain uremic rat serum (URS) for the development 
of in vitro experiments, and the 2 remaining animals submitted to renal ablation were employed for the in vivo detection of ASC, as described later on. At the end of the study period, animals were once more anesthetized with isoflurane and submitted to a xiphopubic laparotomy. The abdominal aorta was punctured, and blood samples were collected to measure serum creatinine $(\mathrm{Scr}, \mathrm{mg} / \mathrm{dL}$ ) and blood urea nitrogen (BUN, mg/dL), using commercially available kits (Creatinina \#35 Kit and Urea CE \# 27 Kit, Labtest, Brazil). The estimated creatinine clearance $(\mathrm{CrCl}, \mathrm{mg} / \mathrm{min})$ was obtained by measuring the urinary creatinine concentration $(\mathrm{Ucr}, \mathrm{mg} / \mathrm{dL})$, with the same colorimetric kit, and performing the following calculations: [(Ucr x UV) / Scr] / 1440. Further corrections for rat body surface area $\left(\mathrm{RBSA} \cong 357 \mathrm{~cm}^{2}\right)$ were obtained by dividing this result by 0.0357 $(\mathrm{CrCl}, \mathrm{mg} / \mathrm{min} / \mathrm{BSA})$.

The left kidney was removed and weighted. For hypertrophy analysis we performed the following calculation $=($ left kidney weight $/$ final body weight $) \times 1000$. Kidney samples were then fractionated, half of it was briefly fixed in Du Boscq-Brasil solution for 30 minutes, followed by fixation with buffered paraformaldehyde ( $\mathrm{pH}: 7.4)$ for 24-72 hours, for further histological and immunohistochemical analysis. The remaining half of the left kidney was quickly frozen in liquid nitrogen and kept at $-80^{\circ} \mathrm{C}$ for further analysis of gene expression.

\section{In vivo Detection of ASC}

In order to verify the homing and stability of ASC in the renal parenchyma, 2 additional animals, submitted to the $5 / 6$ renal ablation model of CKD were subjected to renal subcapsular injection of $2 \times 10^{6}$ labeled ASC, after 15 days of CKD induction. Briefly, the nuclear stain 4-6 diamidino-2-phenylindole dihydrochloride (DAPI; Sigma-Aldrich), was 
added to the culture flasks of ASC in the $4^{\text {th }}$ cell and incubated for 1 hour in a humid oven at $37^{\circ} \mathrm{C}, 5 \% \mathrm{CO}^{2}$. The cells were harvested, counted and inoculated into the rat renal subcapsular space. One of the animals was euthanized after $24 \mathrm{~h}$ of cell inoculation, and the other one was kept until 30 days of CKD induction (15 days after ASC injection). The animals were euthanized by IP injection of a lethal dose of Thiopental $(0.1 \mathrm{~g} / \mathrm{rat})$, kidneys were rapidly frozen and processed in histological sections of $4 \mu \mathrm{m}$, which were fixed in acetone, stained with $0.6 \%$ Evans blue and analyzed using a fluorescence microscope [10].

\section{Histological and Immunohistochemical Analysis}

The fixed renal fragments were dehydrated, diaphanized and included in paraffin blocks from which 4- $\mu$ m-thick tissue sections were obtained. For all histological and immunohistochemical analyses, kidney sections were deparaffinized and rehydrated through a sequence of xylol and alcohol baths.

The presence of glomerular architecture disruption was verified in the renal sections of animals from the different experimental groups, stained by the Periodic Acid-Schiff (PAS) technique. The percentage of glomerulosclerosis (GS\%) was assessed by the blinded analysis of 50 glomeruli of each animal, under a final magnification of $400 x$. The extent of renal cortical interstitial fibrosis was estimated in renal sections stained with Masson's Trichrome. The percentage of interstitial fibrosis was determined by the point-counting technique, in 30 consecutive microscopic fields, under a final magnification of 200x [19]. Immunohistochemistry was employed to detect myofibroblasts, by the presence of the $\alpha$-smooth muscle actin protein ( $\alpha$-SMA), macrophages (CD68), T-lymphocytes (CD3), ZO1 
constitutive glomerular protein, and proliferating cells (PCNA), in the renal samples of experimental animals. After dewaxing, $4 \mu \mathrm{m}$ thick slices were subjected to microwave heating in $\mathrm{pH} 6.0$ citrate buffer for antigen retrieval. For CD68 and $\alpha-S M A$, the immunophosphatase technique was performed. The primary monoclonal mouse anti $\alpha-$ SMA (Sigma, \#A2547) and monoclonal mouse anti-CD68 antibodies (Serotec, \#MCA341R) were used, followed by the secondary biotinylated anti-mouse antibody (Vector \# BA2001). Reactions were developed with Fast Red TR salt (Merk, \#F6760), as previously described [17]. For CD3, PCNA and ZO1, the immunoperoxidase technique was performed, employing the primary polyclonal rabbit anti-CD3 (DAKO \#A4052), monoclonal mouse anti-PCNA (Dako, \#M0879) and polyclonal rabbit anti-ZO-1 (ZYMED 617300) antibodies, respectively. Slides were developed with DAB (Dako, Carpinteria, CA, USA) [Machado, 2008]. Quantitative analysis of immunohistochemistry was performed in a blinded fashion. Renal cortical interstitial infiltration by macrophages and Tlymphocytes, as well as cell proliferation were evaluated by counting the number of positive cells for CD68, CD3 and PCNA, in at least 30 microscopic fields for each animal, under a final 400x magnification. The percentage of renal interstitial area occupied by $\alpha-$ SMA was achieved using the same point-counting technique previously described. Finally, structural integrity of the glomerular filtration barrier was verified through the percentage of glomerular area occupied by ZO-1, in at least 25 glomeruli, under a 400x magnification. 


\section{Gene Expression Analysis}

Quantitative reverse transcription PCR (RT-qPCR) analyses were performed to assess renal cortical expression of genes coding for pro and anti-inflammatory cytokines, such as: IL-1 $\beta, I L-2, I L-4, I L-6$ and IL-10. For this purpose, the total RNA of the renal fragments, previously frozen in liquid nitrogen and kept at $-80{ }^{\circ} \mathrm{C}$, was extracted using Trizol (Ambion Thermo Fischer \#15596018). Constitutive gDNA was eliminated from RNA samples using the Turbo DNAse free ${ }^{T M}$ kit (Invitrogen \#AM1907). RT was carried out using M-MLV RT enzyme (Promega \#M1705) to obtain cDNA. PCR were then conducted using specific pairs of primers (Supplementary Table 1) and the Syber GreenER qPCR Supe Mix Universal (Invitrogen \#11762), in the StepOne Plus equipment (Applied Biosystetems - Life Technologies). The Bact gene, coding for constitutive Beta-actin protein (BACT), was used as an endogenous control of the reactions and the products of the RT-qPCR reactions were quantified by the $\Delta \Delta C$ t relative method. The primer sequences employed in this technique can be found in Supplementary Table 1.

\section{In vitro Experiments}

In order to verify if the treatment with an ARB could somehow impair or reduce the survival of ASC in the renal parenchyma of CKD animals, which would force us to choose another class of RAAS blocker to associate to our experimental cell therapy, we performed in vitro experiments to determine if Losartan would be toxic to these cells. At the $4^{\text {th }}$ cell passage, ASC were seeded in cell plates and cultured until achieving $70 \%$ of confluence, when the complete medium was removed and replaced with serum-deprived medium for $24 \mathrm{~h}$. After this period, cells were finally submitted to the following 
treatments: $15 \%$ of $N R, 15 \%$ of URS or $15 \%$ of URS $+10 \mu \mathrm{M}$ of Losartan, following the literature [20]. Cells were appropriately kept in a humid oven at $37^{\circ} \mathrm{C}, 5 \% \mathrm{CO}_{2}$ for further 24h and then fixed for immunofluorescence analysis.

\section{Statistical Analysis}

Results were presented as mean \pm SE. One-way Analysis of Variance (ANOVA), followed by appropriate Tukey post-test was performed to compare all the groups. All calculations were performed using the GraphPad Prism ${ }^{\circledR}$ software version 7.0, and $p$ values below 0.05 were considered significant [21]. 


\section{RESULTS}

\section{ASC inoculation associated to Losartan, reversed hypertension and reduced the mortality} of animals with CKD.

As expected, animals submitted to the model exhibited significant hypertension after 15 days of CKD induction, when compared to time-paired Sham animals ( $180 \pm 8$ vs. $133 \pm 3$,

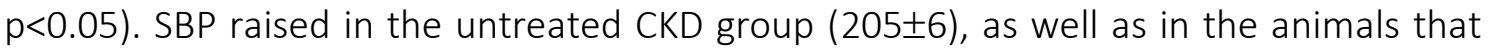
received only the cell therapy (CKD+ASC; 203 18 ), which remained significantly higher than that observed in Sham at 30 days of renal ablation (140 \pm 2$)$, in both of these groups. Losartan treatment promoted a decrease in SBP, which was statistically different in the group CKD+LOS compared to untreated CKD 30d (174 \pm 9 vs. 205 $\pm 6, p<0.05)$. Surprisingly, the association of this pharmacological treatment with the subcapsular ASC inoculation promoted an even more pronounced reduction in arterial hypertension in the CKD+ASC+LOS group, in which SBP values were not statistically different from those of Sham (163 \pm 9 p >0.05 vs.140 \pm 2$)$. The follow-up of SBP in each experimental group, along the study period, can be seen in figure $1 \mathrm{~A}$. To further analyze the impact of the experimental treatments on the evolution of systemic hypertension in the $5 / 6$ model, we calculated the $\triangle S B P$, obtained from the following subtraction: (final SBP, at 30d) - (basal SBP, obtained before the start of treatments, at $15 d$ of CKD induction). This calculation was made individually for each animal, and the mean and standard error values were used in the construction of the graph shown in figure 1B. As observed, the untreated CKD animals had an increase in SBP of approximately $70 \%$ between the $15^{\text {th }}$ and $30^{\text {th }}$ days of analysis, while the monotherapies slowed down this progression: The SBP increase in the CKD+ASC group was around $20 \%$ and in CKD+LOS group, nearly only $5 \%$. Remarkably, the 
ASC+LOS association promoted the reversal of hypertension, as SBP values decreased in CKD+ASC+LOS 30d animals by around 20\% after the treatments.

Accordingly, the percentage of survival among the animals between the $15^{\text {th }}$ and the $30^{\text {th }}$ day after CKD induction followed the dynamics of hypertension in this model. The survival rate was around $90 \%$ in the groups that did not receive Losartan (untreated CKD and CKD+ASC groups), while there was no mortality in the groups CKD+LOS and CKD+ASC+LOS in this same period (100\% of survival), as shown in Supplementary Table 2.

\section{The association of ASC to Losartan promoted the regression of proteinuria and albuminuria in the established CKD model.}

As can be seen in Figure $1 \mathrm{C}$ and $1 \mathrm{E}$, the baseline protein and albumin urinary excretion values of the animals at time zero, before CKD induction, were compatible with physiological levels of normal renal function, and very similar among all the animals included in the study. At 15 days after renal ablation, the animals already exhibited significant proteinuria and albuminuria, when compared to Sham $(81 \pm 20$ vs. $20 \pm 2$, and $51 \pm 19$ vs. $1 \pm 1, p<0.05)$. At this point, rats underwent to the remnant model were subdivided into CKD (untreated), CKD+ASC, CKD+LOS and CKD+ASC+LOS groups, based on similar initial proteinuria and albuminuria values. In untreated CKD animals, both proteinuria (196 \pm 41$)$ and albuminuria (122 \pm 28$)$ increased significantly between day 15 and day 30 after $5 / 6$ renal ablation, reaching approximately twice the value observed at 15 days, at the end of the study (Figure 1D and 1F). The administration of both ASC or LOS monotherapies promoted deceleration of the progression of proteinuria and albuminuria in CKD+ASC (118 \pm 30 and $63 \pm 20)$ and CKD+LOS (110 \pm 46 and $71 \pm 37)$, 
respectively. Surprisingly, CKD+ASC+LOS animals exhibited significant regression of both

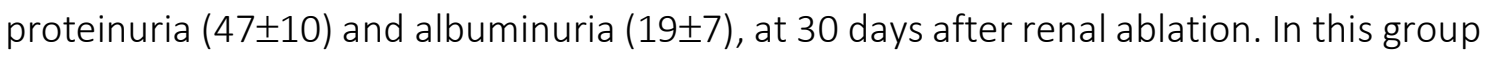
the final values of protein and albumin urinary excretion were not statistically different from those observed in the Sham group.

Detection of ASC in the renal parenchyma of CKD rats after 15 days of renal subcapsular inoculation.

As illustrated in the upper part of figure 2,15d after CKD induction, 2 animals received a subcapsular injection of $2 \times 10^{6} \mathrm{ASC}$, previously labeled with DAPI (2A). One of these rats was euthanized after $24 \mathrm{~h}$ of ASC inoculation, and the other one was kept for more 15 days after cell therapy. Evans blue-stained renal slides were analyzed under fluorescence microscope, as represented in the illustrative microphotographs in $2 \mathrm{~B}$. As shown, DAPIlabeled ASC were easily detected in the renal parenchyma of CKD animals after 24h and $15 \mathrm{~d}$ of subcapsular inoculation (fluorescent blue cells).

ASC inoculation combined to Losartan treatment averted the progression of structural glomerular damage in experimental CKD.

Glomerular architecture was evaluated in PAS-stained renal sections, under a final 400x magnification, as illustrated in figure $3 \mathrm{~A}$. The percentage of glomerulosclerosis (GS\%) in each experimental group was represented as a bar graph, shown in figure 3B. Corroborating previous findings, animals submitted to the $5 / 6$ renal ablation model already presented a numerically high GS\% after 15 days of CKD induction (21 \pm 5$)$, which markedly progressed over time, achieving statistically significant values at 30 days of CKD in both untreated and CKD+LOS groups, when compared to time-paired Sham rats ( $28 \pm 6$ 
and $23 \pm 6$ vs. $3 \pm 1, p<0.05$, respectively). ASC inoculation alone $(17 \pm 5)$ or in association to Losartan (13 \pm 2$)$ significantly prevented GS progression in CKD+ASC and CKD+ASC+LOS groups, which did not differ statistically from control group.

We further analyzed the percentage of glomerular area occupied by the constitutive protein ZO1, related to the integrity of the glomerular filtration barrier. Illustrative microphotographs of renal sections submitted to immunohistochemistry for ZO1 can be seen in figure 3C, ZO1\% bar graphs, in figure 3D. All CKD animals exhibited reduced percentage of the glomerular area occupied by ZO1, when compared to Sham; except for the rats treated with $\mathrm{ASC}+\mathrm{LOS}$ association, in which ZO1\% was statistically similar to that observed in Sham animals ( $53 \pm 3$ vs. $52 \pm 4$ p>0.05).

\section{Subcapsular ASC inoculation was as effective as Losartan in attenuating the renal fibrosis} in the remnant kidney model.

Renal cortical interstitial fibrosis was evaluated in Masson's trichrome sections by the presence of interstitial collagen, stained in blue, under a final 200x magnification, as illustrated in figure 4A. Moreover, immunohistochemistry for $\alpha$-SMA was employed to access the presence of renal interstitial myofibroblasts, major effector cells of fibrogenesis, stained in red, under a final 200x magnification, as shown in figure 4C. The percentage of interstitial fibrosis (INT\%) and renal interstitial area occupied by $\alpha$-SMA, in each experimental group, were represented as bar graphs, shown in figures 4B and 4D, respectively. At 15 days of renal ablation, basal CKD animals already showed a significant increase in the renal cortical interstitial area occupied by collagen, as well as in the fraction of the cortical interstitium occupied by $\alpha$-SMA, when compared 
to the control group ( $2.0 \pm 0.3$ and $3.6 \pm 0.7$ vs. $1.1 \pm 0.2$ and $1.0 \pm 0.1, p<0.05)$, characterizing the presence of interstitial fibrosis, which persisted after 30 days of CKD induction in untreated rats $(5.7 \pm 1.5$ and $3.5 \pm 0.5$ vs. $1.1 \pm 0.2$ and $1.0 \pm 0.1, p<0.05)$. Animals treated between 15 and $30^{\text {h }}$ days of CKD with LOS or ASC monotherapies or with ASC+LOS association, showed a numerical reduction of both INT\% and interstitial $\alpha$-SMA which was equivalent among all treated groups.

The association of ASC to Losartan interrupted the progression of renal cortical inflammation and downregulated IL- 6 gene overexpression in the $5 / 6$ ablation model of CKD.

Interstitial inflammation is one of the main features of CKD progression. Here we evaluated this process by both the presence and intensity of renal infiltration by leukocytes and by the increased proliferation of interstitial cells in the renal parenchyma. Illustrative microphotographs of immunohistochemistry for CD68+ Macrophages (5A), CD3+ T-Lymphocytes (5C) and PCNA+ interstitial cells (5E) are presented in figure 5. As can be seen in the bar graphs in figures 5B, 5D and 5F, after 15 days of CKD induction, animals already presented marked macrophage infiltration and significant increase in the presence of T-lymphocytes and PCNA+ interstitial cells, when compared to the control animals $(93 \pm 18,53 \pm 10$ and $101 \pm 16$ vs. $36 \pm 7,14 \pm 3$ and $24 \pm 3$, respectively). Renal inflammation progressed over time in untreated rats, reaching statistically significant values after 30 days of CKD in all analyzed parameters: (153 \pm 35 for CD68, 132 \pm 30 for CD3 and $135 \pm 27$ for PCNA vs. $36 \pm 7,14 \pm 3$ and $24 \pm 3$, respectively, $p<0.05$ ). ASC or Losartan monotherapies were not able to detain the progression of renal inflammation in the ablation model. However, the association of ASC+LOS promoted significant 
reduction of macrophage infiltration ( $56 \pm 7$ vs. $153 \pm 35 p<0.05)$, and a relevant reduction in both interstitial lymphocytes ( $76 \pm 12$ vs. $132 \pm 30)$ and PCNA+ cells ( $64 \pm 14$ vs. $135 \pm 27)$. The results of our RT-qPCR analyses of the local renal expression of some of the main pro and anti-inflammatory genes related to CKD progression can be seen as bar graphs in figure 6. After 15 days of CKD induction, rats already exhibited IL-1 $\beta$, IL-2, IL-4, IL-6, IL-10 and TGF- $\beta$ overexpression, which remained up to 30 days of renal ablation. It is noteworthy that the association of ASC to Losartan promoted further upregulation of IL$4(6 C)$ and significant downregulation of IL-6 (6D) gene expressions.

\section{Losartan improved cell viability and proliferation of ASC in vitro.}

As illustrated in Supplementary Figure 3, cultured ASC stimulated with Losartan in vitro exhibited a significant increase in the total number of living cells $/ \mathrm{mm}^{2}$, as well as in the number of proliferating ASC, which were positive for PCNA in immunofluorescence analysis. 


\section{FIGURES}

\section{FIGURE 1}

A

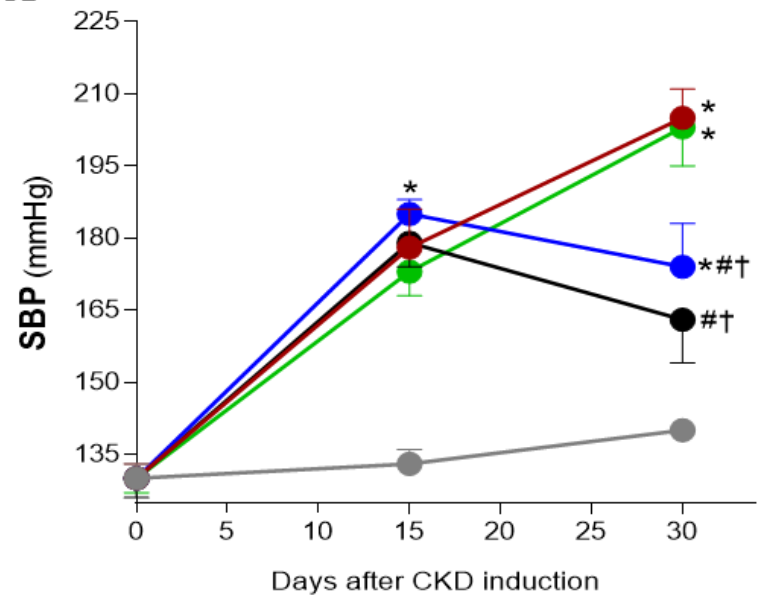

C

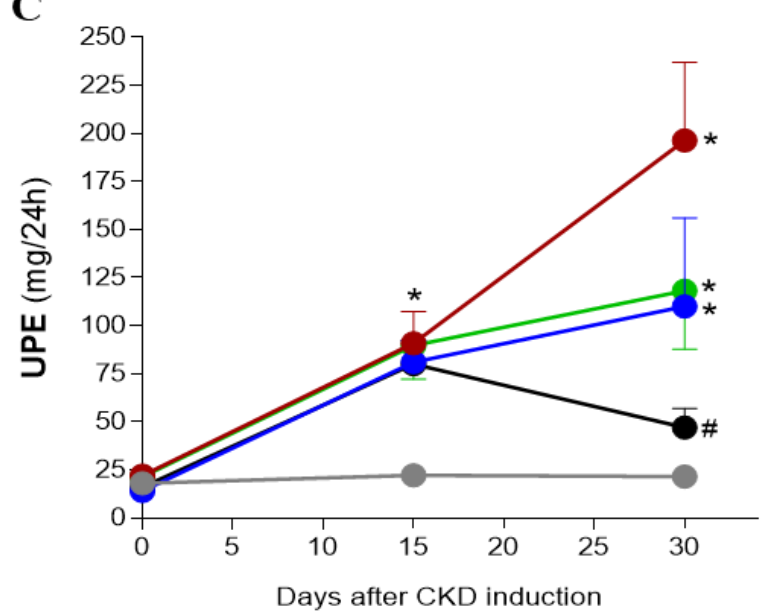

E

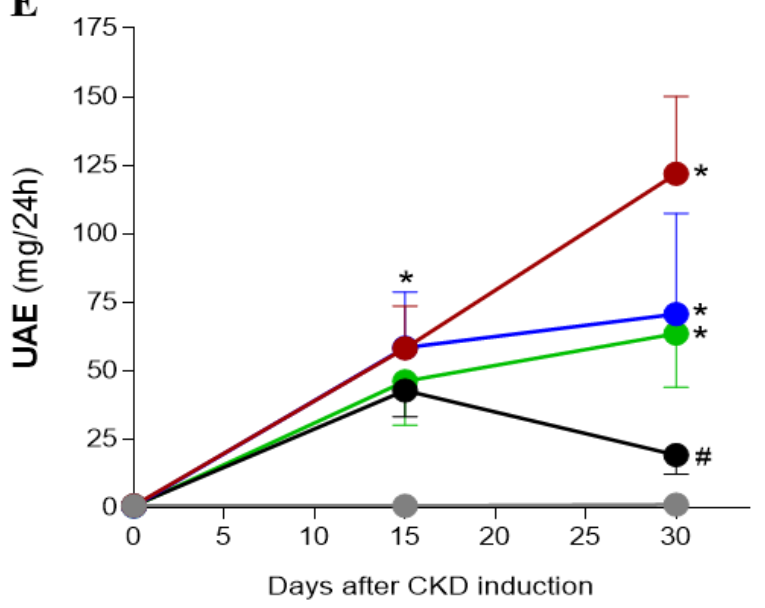

B

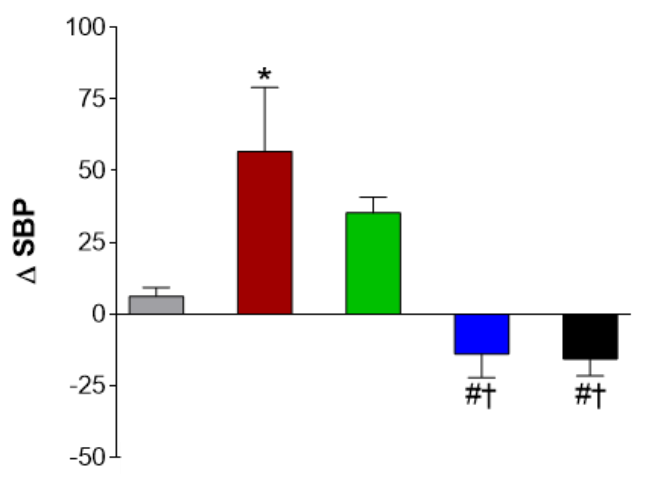

D

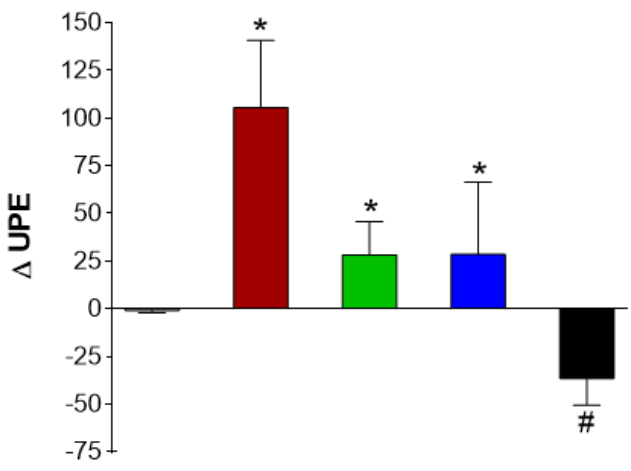

$\mathbf{F}$

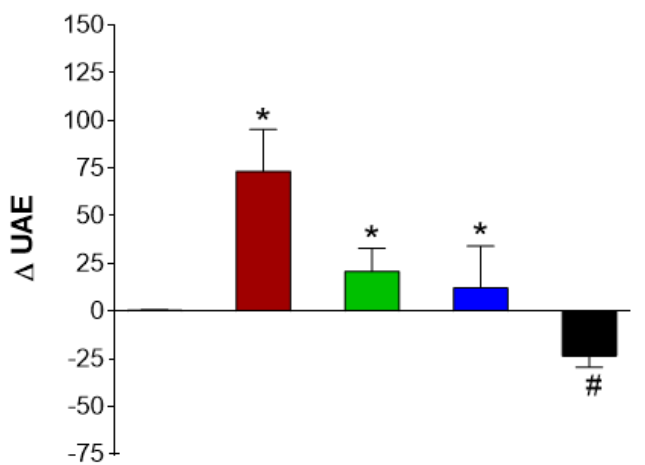

Sham

CKD

CKD+ASC

CKD+LOS

CKD+ASC+LOS 
FIGURE 2

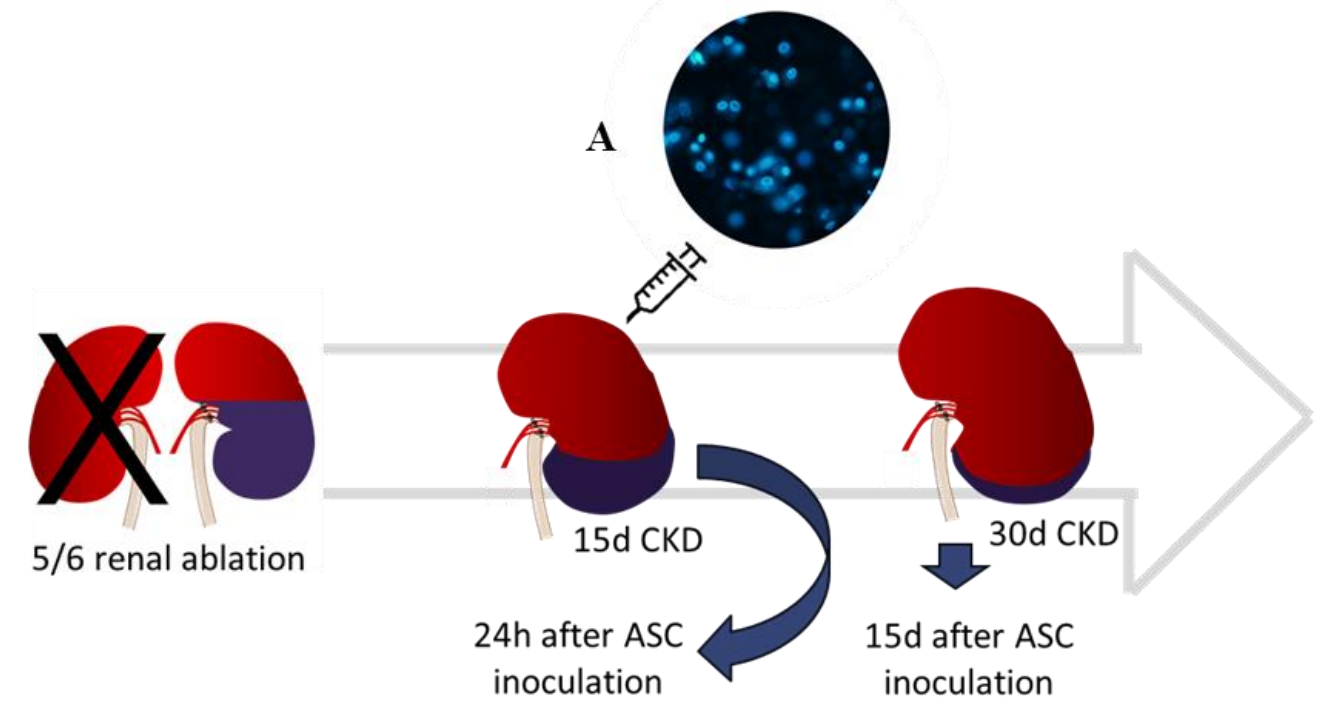

B

Evans Blue
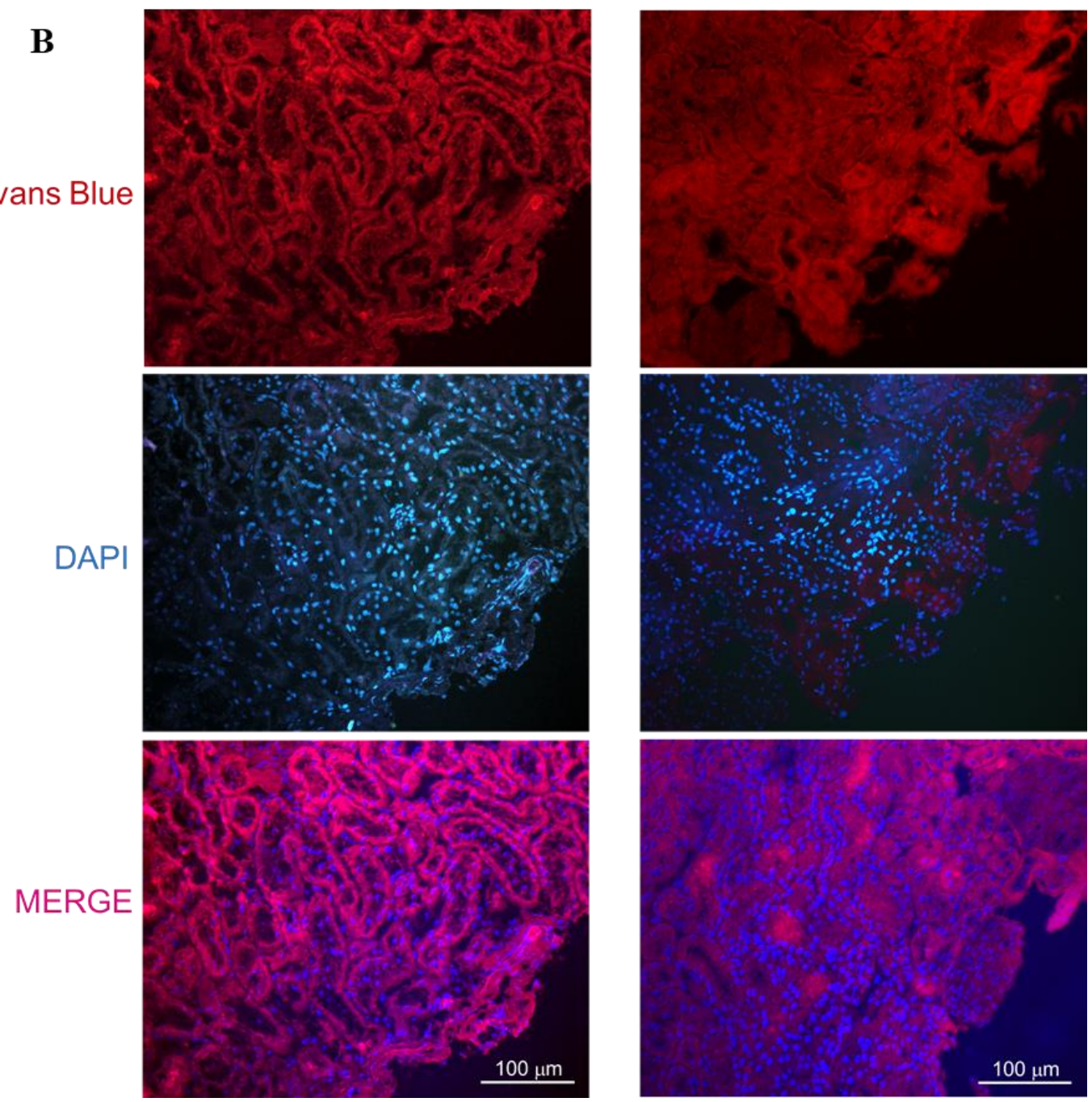


\section{FIGURE 3}

A

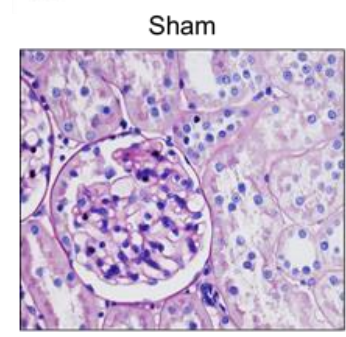

CKD+ASC

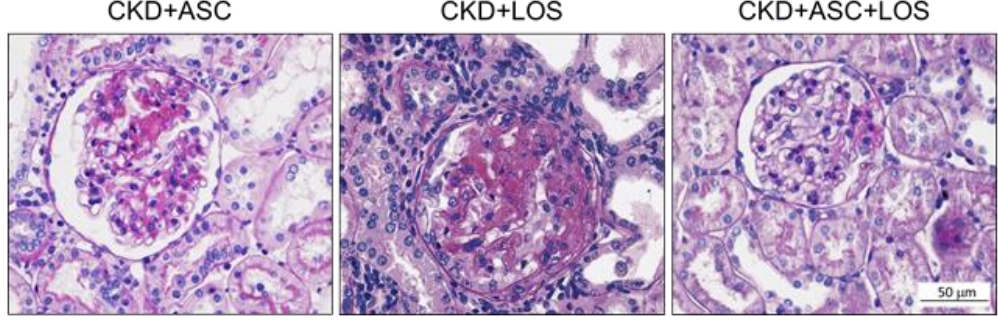

C

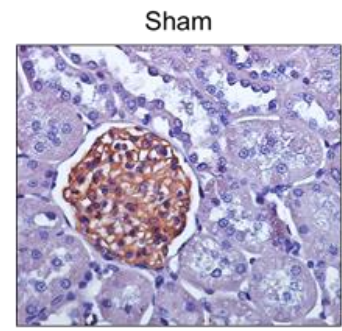

CKD+ASC

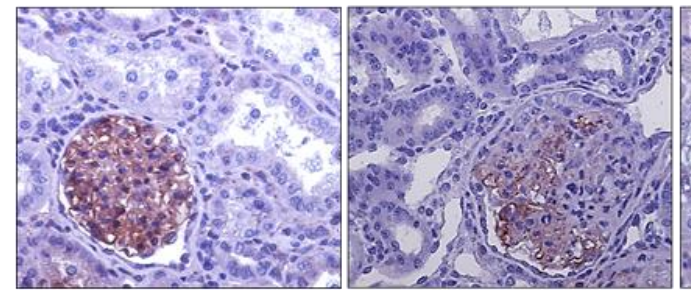

basal CKD

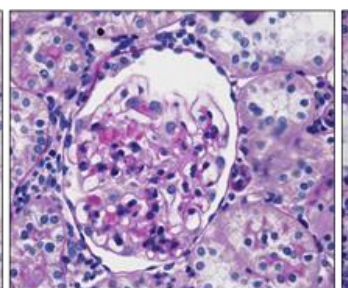

CKD+LOS
CKD

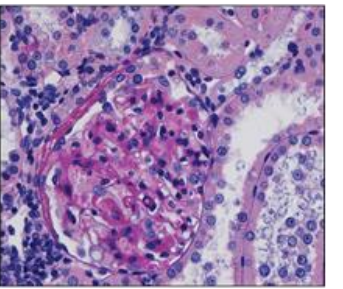

(

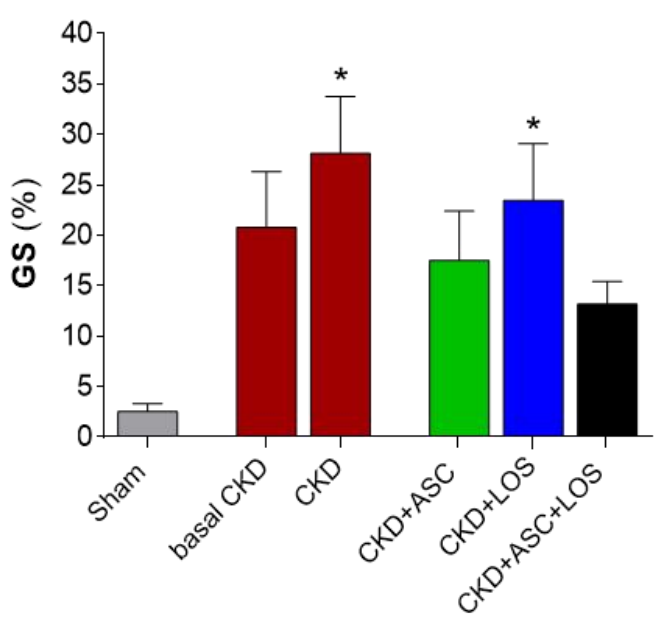

D

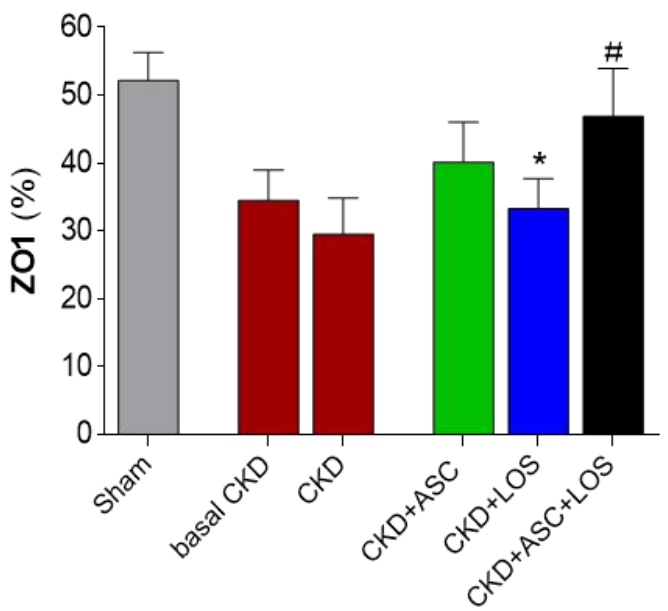




\section{FIGURE 4}

A

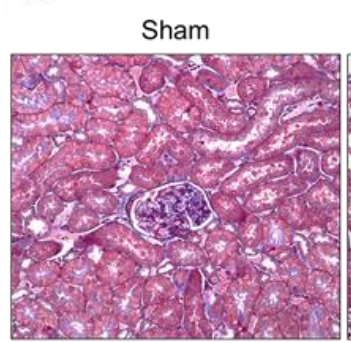

CKD+ASC
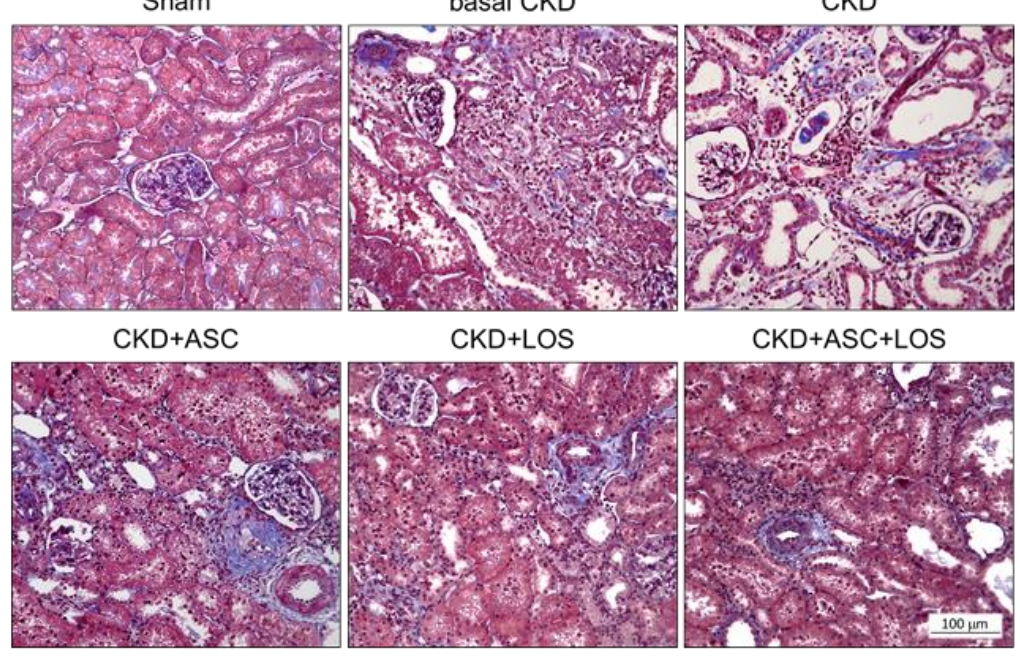

CKD+LOS

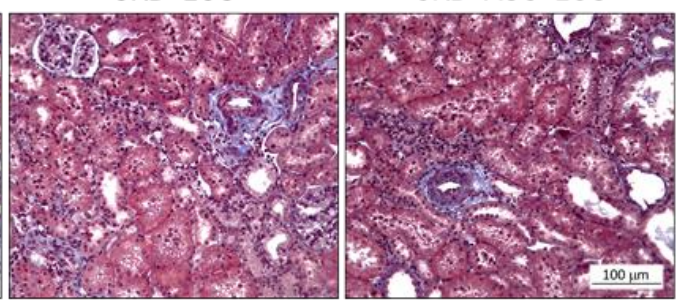

C

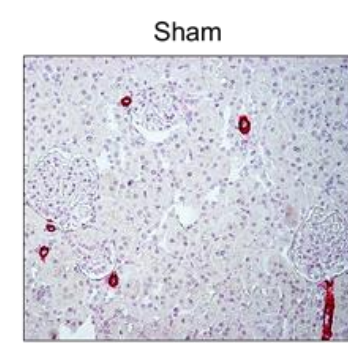

CKD+ASC

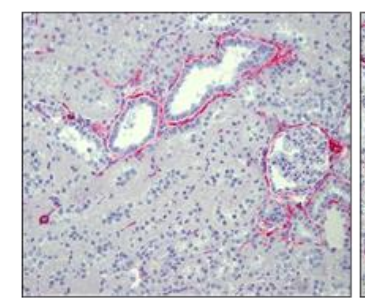

basal CKD

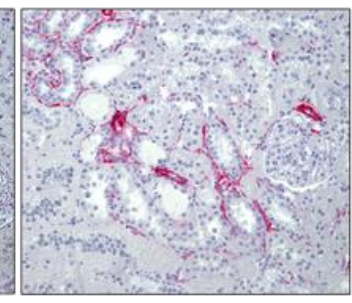

CKD+LOS

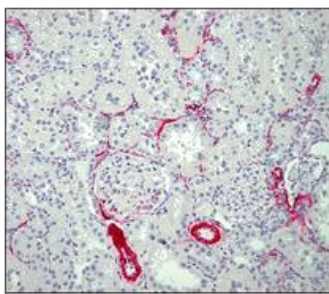

B

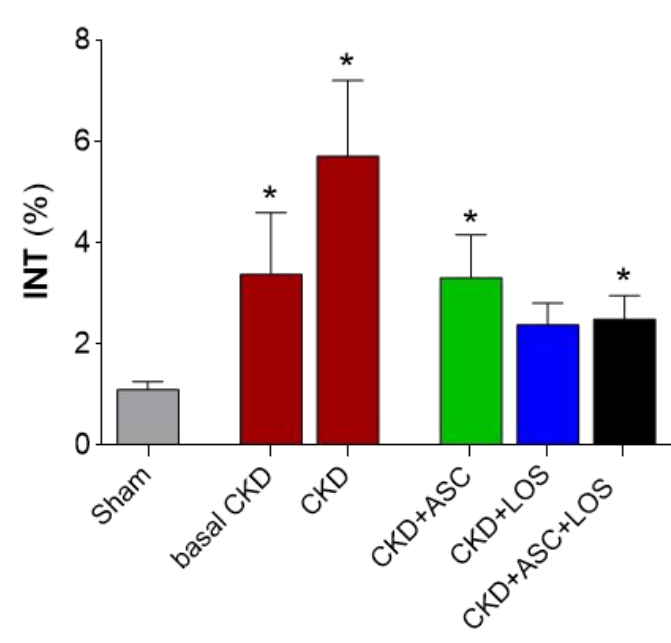

D

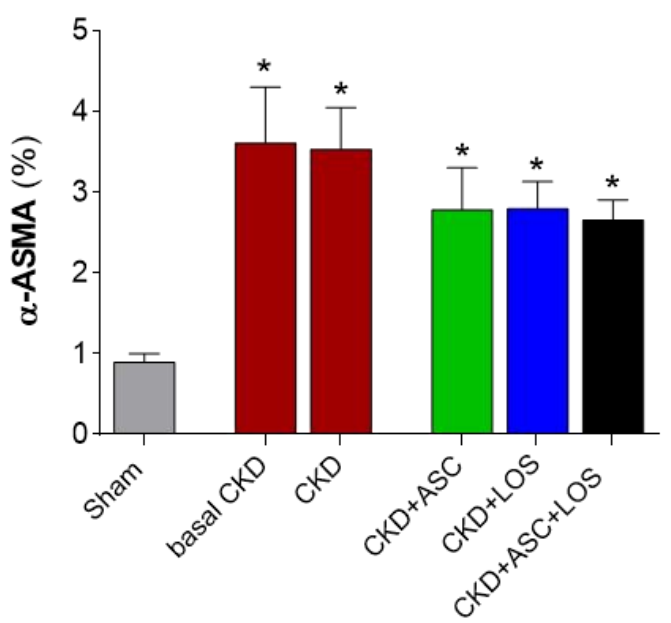




\section{FIGURE 5}

A

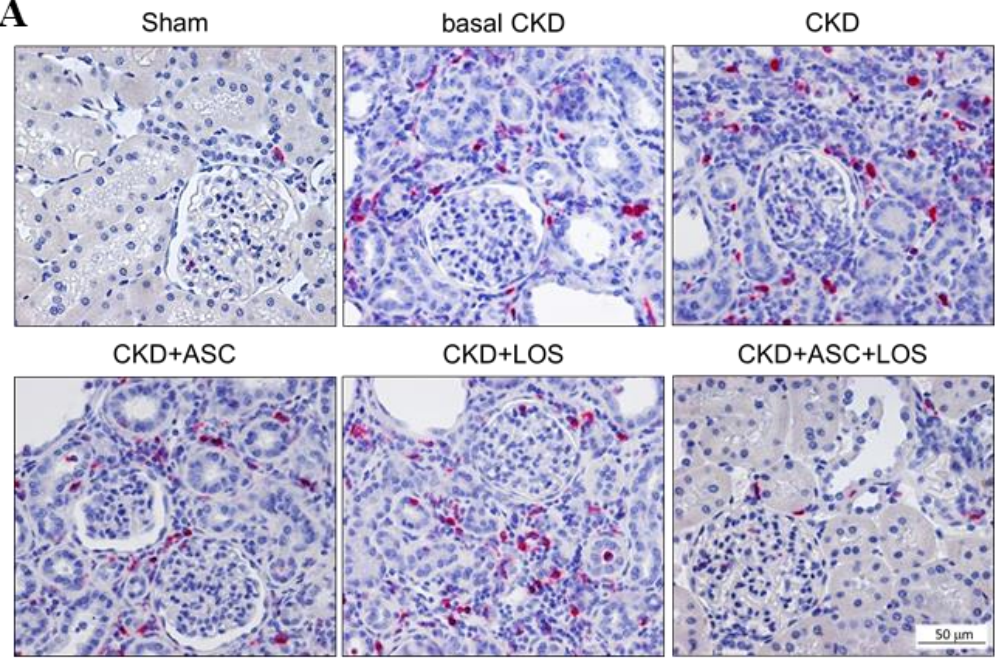

C

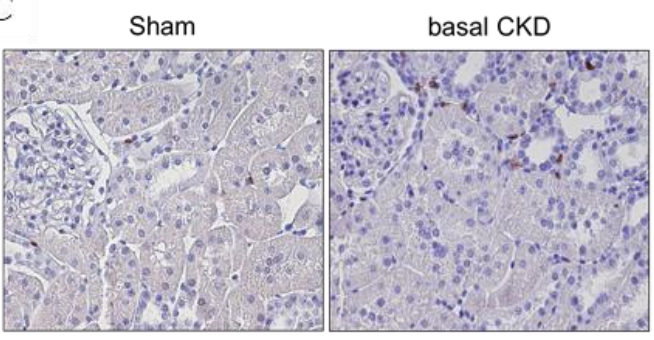

CKD+ASC
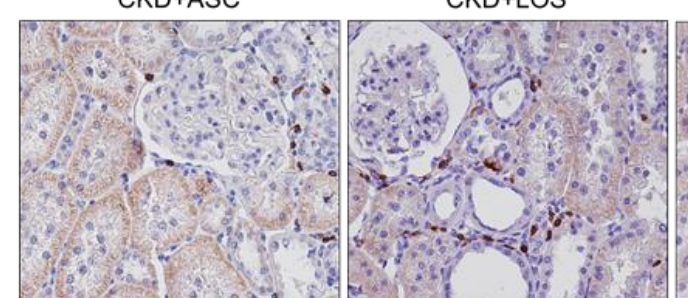

$\mathbf{E}$

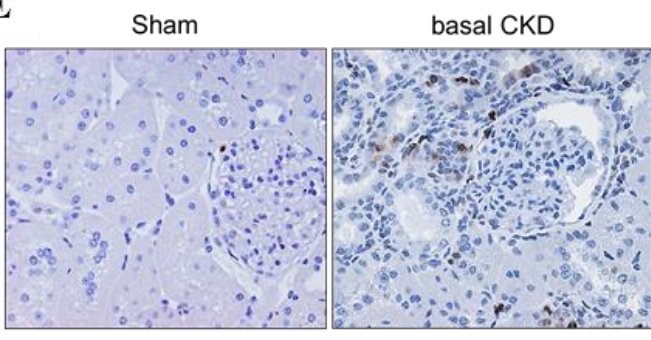

CKD+ASC

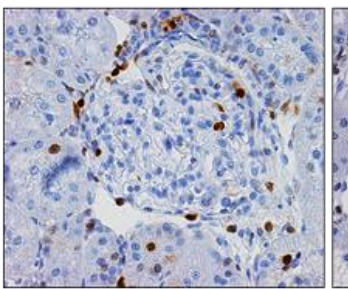

CKD+LOS

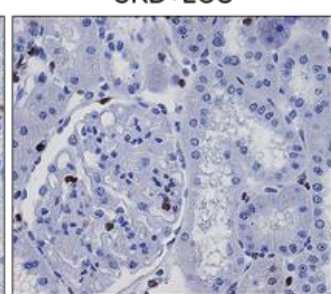

CKD

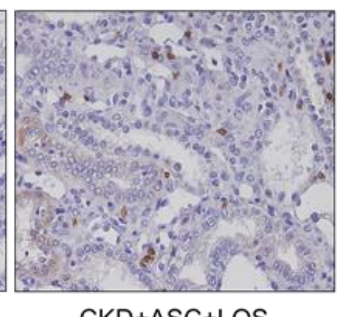

CKD+ASC+LOS
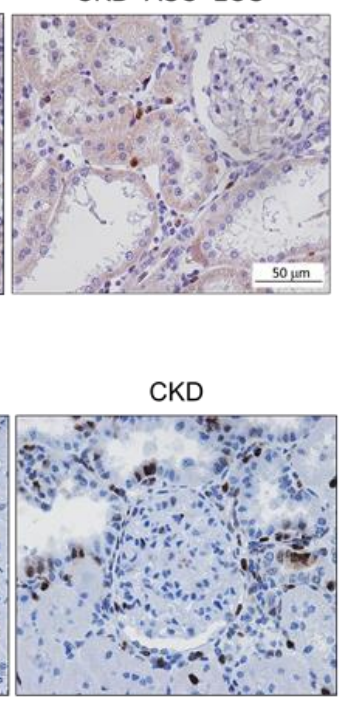
CKD+ASC+LOS

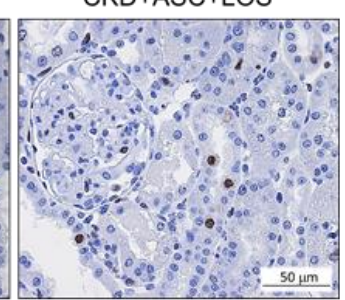

B

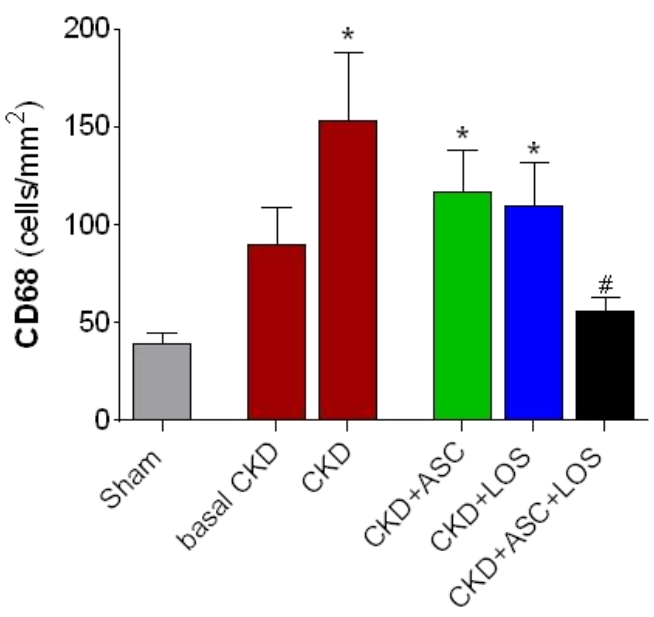

D

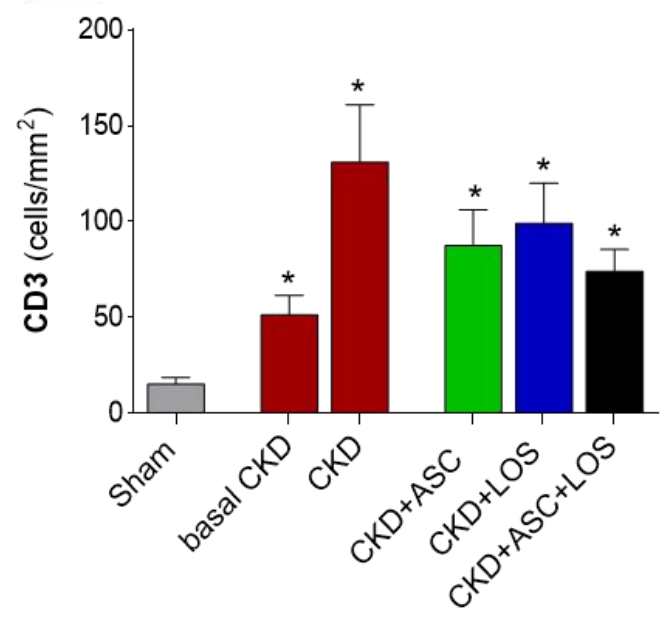

F

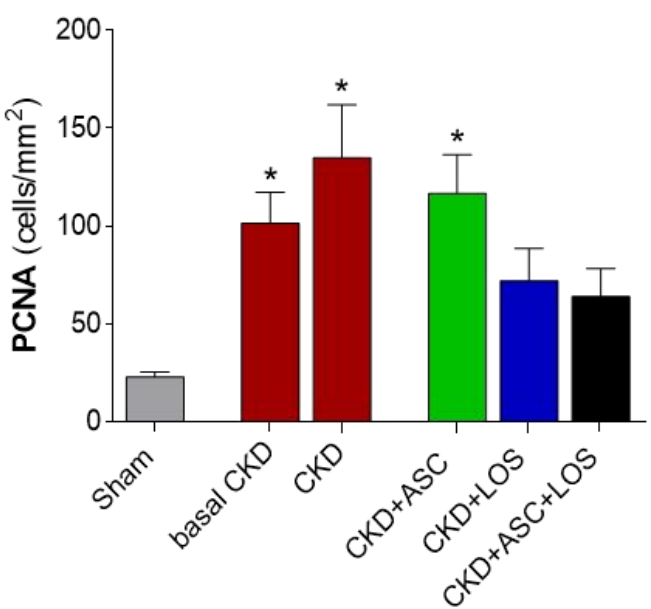




\section{FIGURE 6}

A

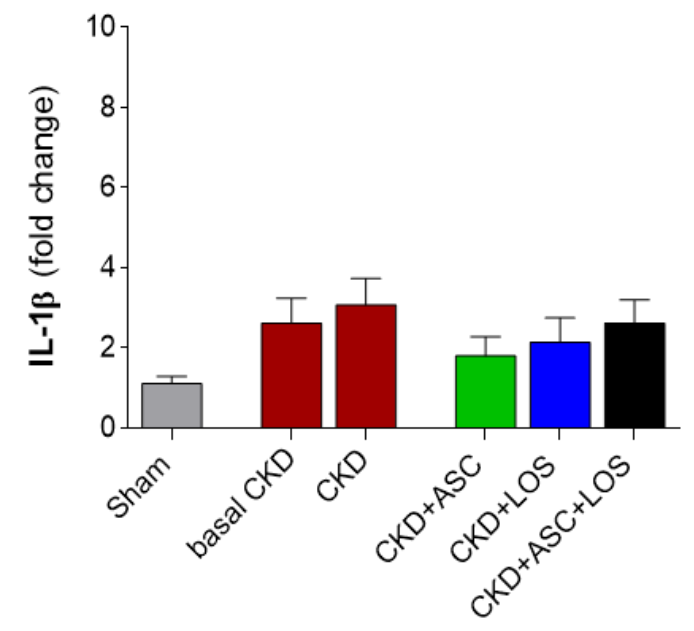

C

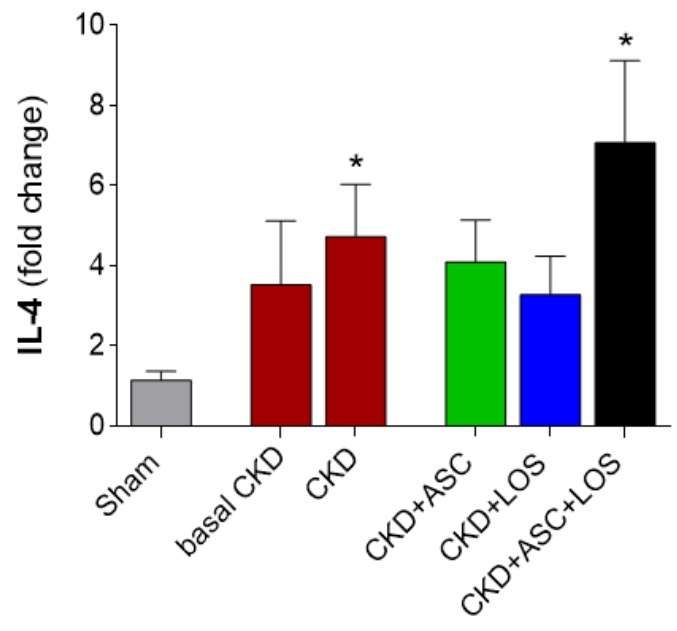

$\mathbf{E}$

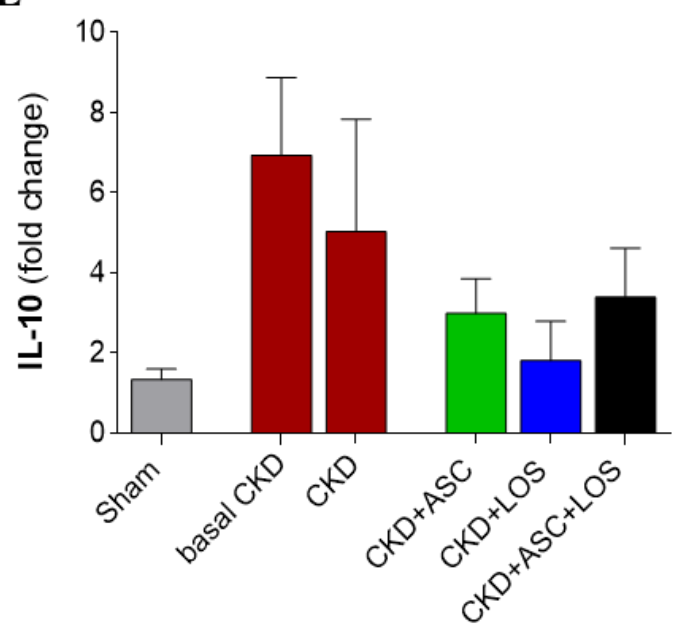

B
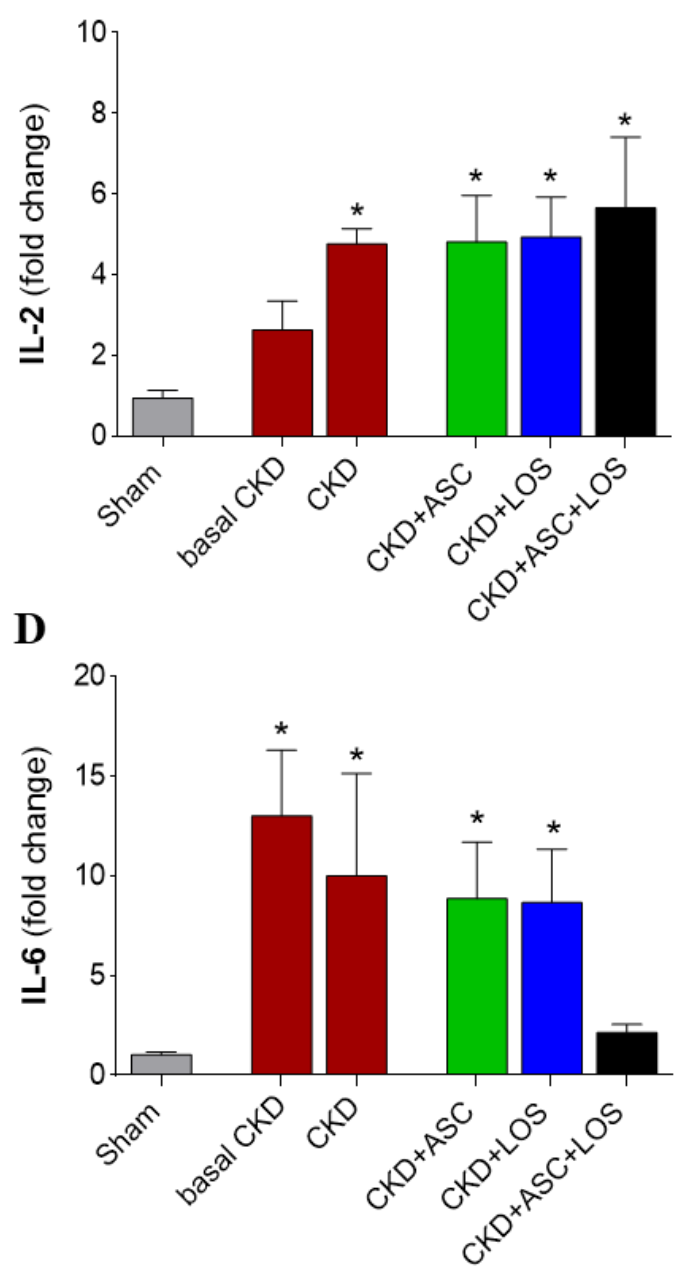

$\mathbf{F}$

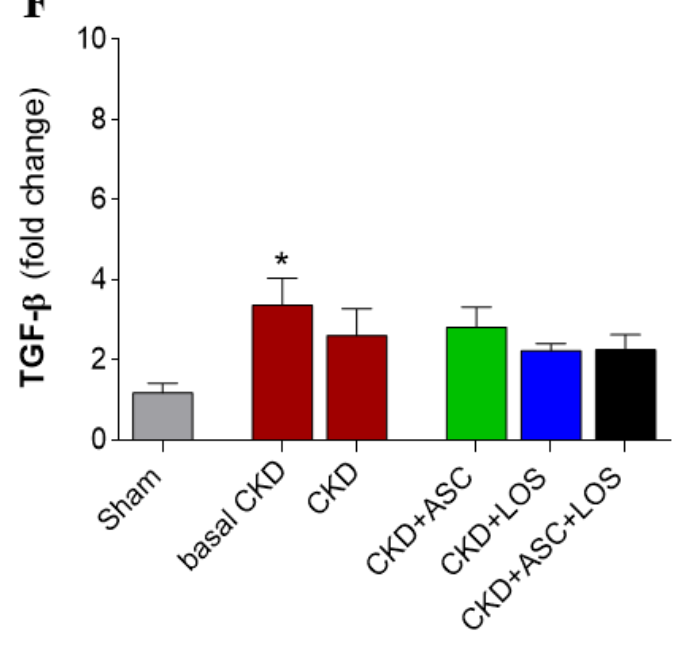




\section{FIGURE LEGENDS}

Figure 1: Systolic blood pressure (SBP, $\mathrm{mmHg}$ ), urinary protein (UPE, $\mathrm{mg} / 24 \mathrm{~h}$ ) and albumin (UAE, mg/24h) excretion of the animals of each experimental group: (A) SBP, (C) UPE, (E) UAE time-course graphs. (B) SBP, (D) UPE, (F) UAE delta bar-graphs, obtained by subtracting the values observed at 30 days from those obtained at 15 days, before the beginning of treatments. Statistical differences are: ${ }^{*} p<0.05$ vs. Sham, $\# p<0.05$ vs. CKD, ${ }^{\dagger} p<0.05$ vs. CKD+ASC, $\neq p<0.05$ vs. CKD+LOS, in the respective time points.

Figure 2: In vivo detection of ASC: After 15d of CKD induction, animals received a subcapsular injection of previously DAPI-labeled ASC (A), which could be detected $24 \mathrm{~h}$ and also $15 \mathrm{~d}$ after inoculation, under a final 200x magnification, using a fluorescence microscope (B).

Figure 3: Glomerular architecture: (A) Illustrative microphotographs of PAS stained sections of the animals of each experimental group, at the end of the study. (B) Bar graphs of the percentage of glomerulosclerosis (GS) in the animals included in the protocol. (C) Illustrative microphotographs of immunohistochemistry for ZO1 in each experimental group. (D) Bar graphs of the percentage of glomerular area occupied by ZO1 in the animals included in each experimental group, at the end of the study. Statistical differences are: ${ }^{*} p<0.05$ vs. Sham, $\S p<0.05$ vs. basal $C K D, \# p<0.05$ vs. CKD, $\uparrow p<0.05$ vs. CKD+ASC, $\neq p<0.05$ vs. $C K D+L O S$.

Figure 4: Interstitial fibrosis and myofibroblasts infiltration. (A) Illustrative microphotographs of Masson's Trichrome staining in the experimental groups. (B) Bar graphs representing the percentage of the renal interstitial area occupied by fibrosis in the studied animals. (C) Microphotographs illustrating the immunohistochemistry for $\alpha$-ASMA to determine the 
presence of interstitial myofibroblasts in the renal parenchyma of the studied animals. (D) Bar graphs representing the percentage of interstitial area occupied by $\alpha$-ASMA. Statistical differences are: ${ }^{*} p<0.05$ vs. Sham, $\S p<0.05$ vs. basal $C K D, \# p<0.05$ vs. $C K D,+p<0.05$ vs. CKD+ASC, $\neq p<0.05$ vs. CKD+LOS.

Figure 5: Local renal inflammation: Illustrative microphotographs of immunohistochemistry for (A) macrophages (CD68), (C) T-cells (CD3) and (E) Proliferating interstitial cells (PCNA), in each experimental group. Bar graphs representing the number of (B) macrophages, (D) Tlymphocytes and (F) PCNA (cells/mm2) in the renal cortical interstitial area. Statistical differences are: ${ }^{*} p<0.05$ vs. Sham, $\S p<0.05$ vs. basal $C K D, \# p<0.05$ vs. $C K D,+p<0.05$ vs. CKD+ASC, $\neq p<0.05$ vs. CKD+LOS.

Figure 6: Bar graphs depicting the gene expression results of the following pro-inflammatory and anti-inflammatory genes using the RT qPCR technique: (A) IL-1B, (B) IL-2, (C) IL-4, (D) IL-6, (E) IL-10 and (F) TGF- $\beta$. Statistical differences are: ${ }^{*} p<0.05$ vs. Sham, $\S p<0.05$ vs. basal CKD, $\# p<0.05$ vs. $C K D, \uparrow p<0.05$ vs. CKD+ASC, $\neq p<0.05$ vs. $C K D+L O S$. 


\section{SUPPLEMENTARY FIGURE 1}

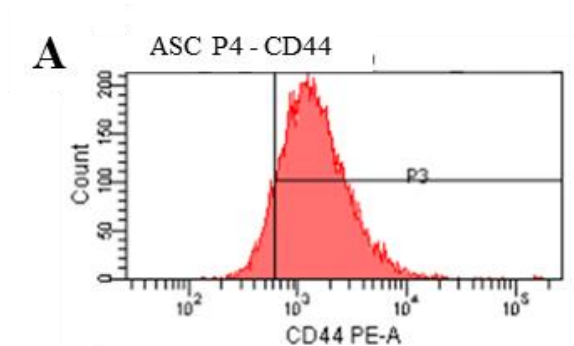

C

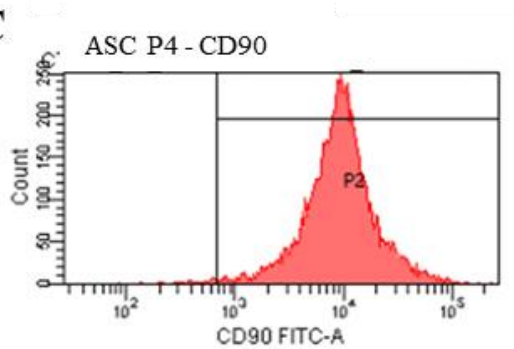

$\mathbf{E}$

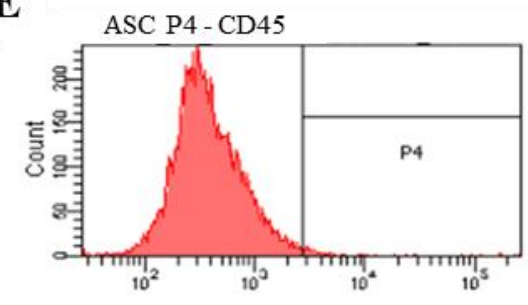

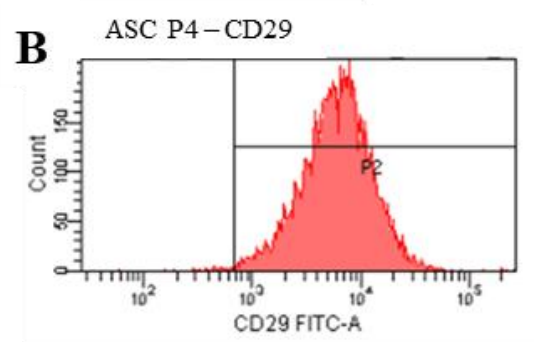

D

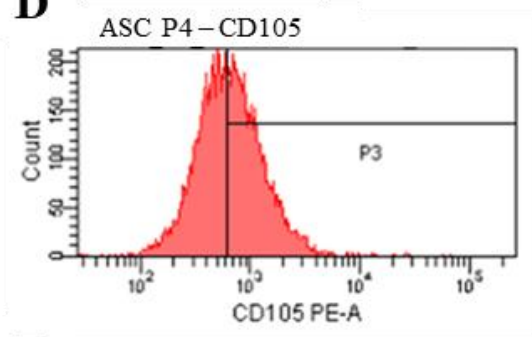

F

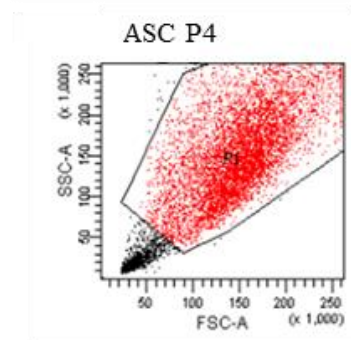

G

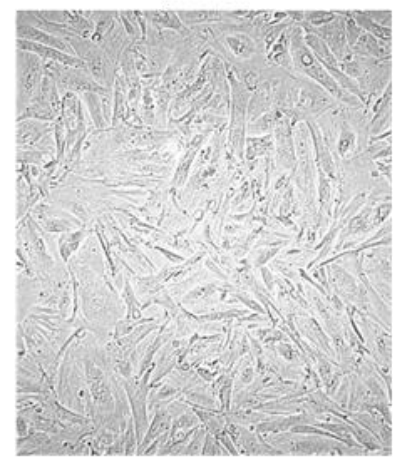

$\mathbf{H}$

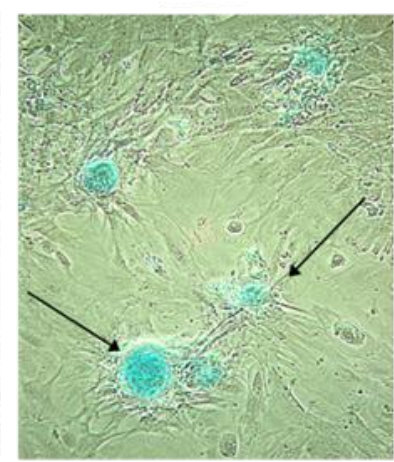

$\mathbf{J}$
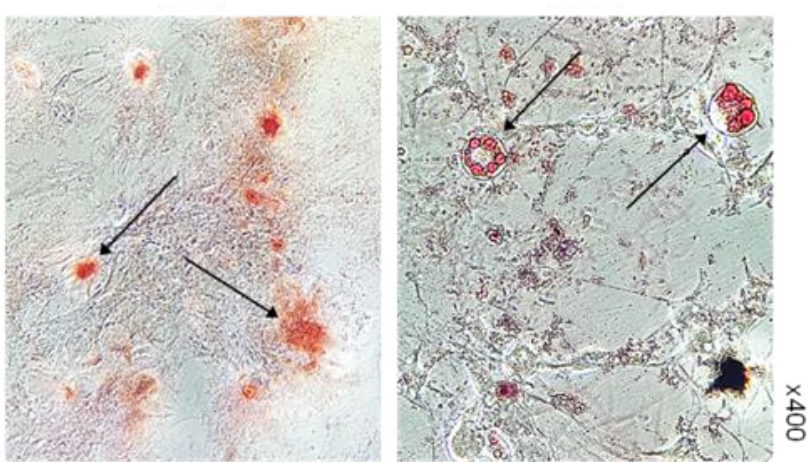


\section{SUPPLEMENTARY FIGURE 2}

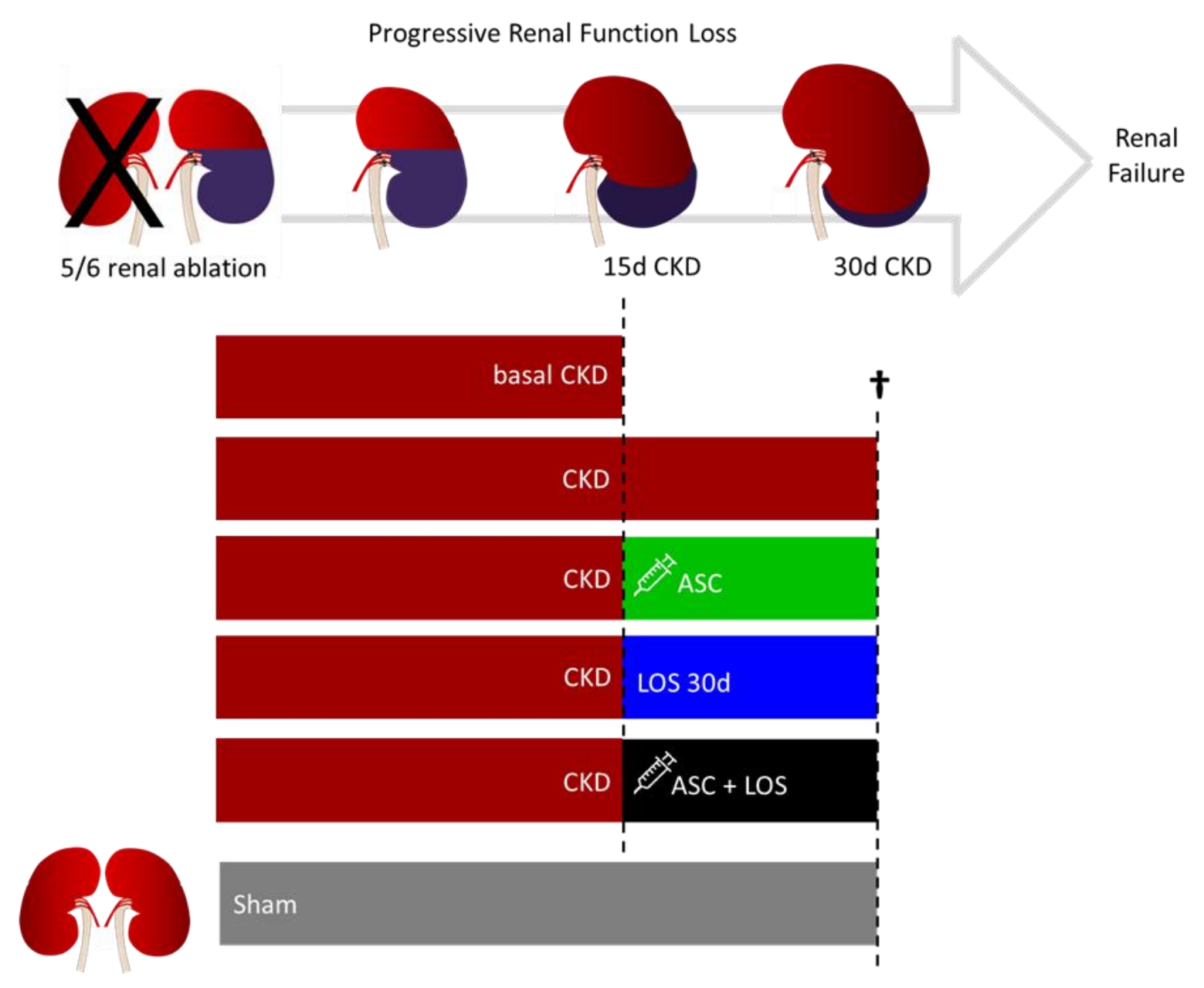




\section{SUPPLEMENTARY FIGURE 3}

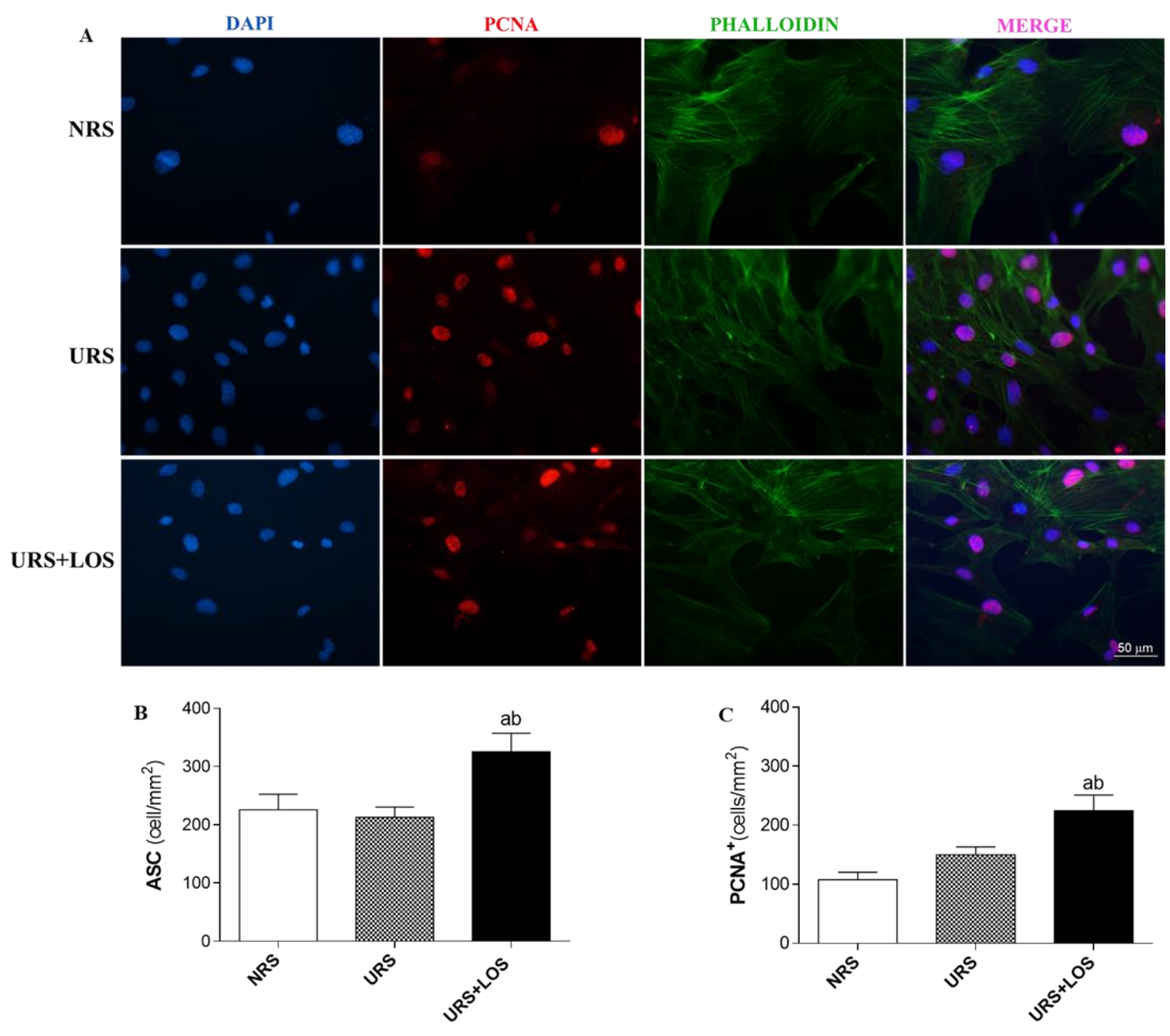




\begin{tabular}{ccc}
\hline GENE & FORWARD & REVERSE \\
\hline $\boldsymbol{B a c t}$ & AGGGAAATCGTGCGTGACAT & CCATACCCAGGAAGGAAGGC \\
$\boldsymbol{I l \boldsymbol { l }}$ & ACTGAACTTCGGGTGATCG & GCTTGGTGGTTTGCTACGAC \\
$\boldsymbol{I l \boldsymbol { l }}$ & CCAAGCAGGCCACAGAATTG & CAAATCCAACACACGCTGCA \\
$\boldsymbol{I l}$ & GGAGAACGAGCTCATCTGCA & GGTGCAGCTTCTCAGTGAGT \\
$\boldsymbol{I l \boldsymbol { l }}$ & CCATCTGCCCTTCAGGAACA & ACTGGCTGGAAGTCTCTTGC \\
$\boldsymbol{I l l 0}$ & GCTCAGCACTGCTATGTTGC & TGTTGTCCAGTCGGTCCTTC \\
\hline
\end{tabular}




\section{SUPPLEMENTARY TABLE 2}

\begin{tabular}{|c|c|c|c|c|c|c|}
\hline & $\begin{array}{c}\text { Sham 30d } \\
\qquad(\mathrm{N}=11)\end{array}$ & $\begin{array}{c}\text { basal CKD } \\
(\mathrm{N}=11)\end{array}$ & $\begin{array}{l}\text { CKD } \\
(\mathrm{N}=15)\end{array}$ & $\begin{array}{c}\text { CKD+ASC } \\
(\mathrm{N}=15)\end{array}$ & $\begin{array}{c}\text { CKD+LOS } \\
(\mathrm{N}=12)\end{array}$ & $\begin{array}{c}\text { CKD }+ \text { ASC }+ \text { LOS } \\
(\mathrm{N}=14)\end{array}$ \\
\hline Survival Rate (\%) & $100 \%$ & - & $92 \%$ & $93 \%$ & $100 \%$ & $100 \%$ \\
\hline BW (g) & $356 \pm 12$ & $276 \pm 9^{\star}$ & $304 \pm 6^{\star}$ & $286 \pm 11^{*}$ & $289 \pm 9^{\star}$ & $296 \pm 5^{\star}$ \\
\hline $\mathbf{U V}(\mathrm{mL})$ & $19 \pm 2$ & $38 \pm 39^{*}$ & $43 \pm 4^{\star}$ & $39 \pm 4^{*}$ & $42 \pm 5^{\star}$ & $38 \pm 3^{*}$ \\
\hline $\operatorname{Scr}(\mathrm{mg} / \mathrm{dL})$ & $0.65 \pm 0.03$ & $0.91 \pm 0.12$ & $0.97 \pm 0.11$ & $1.17 \pm 0.15^{\star}$ & $0.82 \pm 0.06$ & $0.83 \pm 0.06$ \\
\hline $\operatorname{Ucr}(\mathrm{mg} / \mathrm{dL})$ & $65 \pm 8$ & $21 \pm 4^{\star}$ & $24 \pm 3^{\star}$ & $25 \pm 4^{\star}$ & $30 \pm 5^{\star}$ & $26 \pm 2^{\star}$ \\
\hline CrCl (mg/min/RBSA) & $31 \pm 3$ & $20 \pm 6$ & $23 \pm 5$ & $16 \pm 2^{*}$ & $35 \pm 10$ & $24 \pm 2$ \\
\hline BUN (mg/dL) & $51 \pm 4$ & $105 \pm 13^{\star}$ & $88 \pm 8^{\star}$ & $101 \pm 15^{\star}$ & $84 \pm 7^{\star}$ & $82 \pm 4^{\star}$ \\
\hline Renal Hypertrophy & $3.4 \pm 0.1$ & $5.0 \pm 0.3^{\star}$ & $5.2 \pm 0.2^{\star}$ & $4.9 \pm 0.5^{\star}$ & $5.3 \pm 0.4^{\star}$ & $4.6 \pm 0.1^{\star}$ \\
\hline
\end{tabular}




\section{SUPPLEMENTARY FIGURE AND TABLE LEGENDS}

Supplementary Figure 1: Characterization of ASC at the 4th passage was performed by flow cytometry and cell plasticity tests: Cell population was positive for typical mSC biomarkers: CD44 (A), CD29 (B), CD90 (C) and CD105 (D), and negative for the pan leukocyte marker CD45 (E). 10,000 events were analyzed, of which 8,898 were viable (F). ASC phenotype before the plasticity tests is shown in $(\mathrm{G})$. Chondrogenic differentiation was evidenced by the presence of sulfated matrix proteoglycans, highlighted in turquoise blue and stained with Alcian blue $(\mathrm{H})$; the osteogenic differentiation was verified by means of reddish calcium crystals stained with Alizarin Red (I) and finally, the adipogenic differentiation was verified by the presence of lipid droplets stained in red by Oil-red $O(J)$. Microphotographs were taken under 400x magnification.

Supplementary Figure 2: Experimental Protocol. On the 15th day after renal ablation, animals submitted to CKD induction were distributed into five experimental groups, randomized according to their basal UPE, as follows: basal CKD ( $N=11)$, euthanized 15 days after $5 / 6$ renal ablation, $\mathrm{CKD}(\mathrm{N}=13)$, kept untreated for 30 days, $\mathrm{CKD}+\mathrm{ASC}(\mathrm{N}=15)$, that received a subcapsular injection of ASC at the 15th day of CKD and were followed until the 30th day after CKD induction, CKD+LOS ( $\mathrm{N}=12)$, that received $50 \mathrm{mg} / \mathrm{Kg} /$ day of Losartan, diluted in drinking water, from the $15^{\text {th }}$ to the $30^{\text {th }}$ day after renal ablation, and CKD+ASC+LOS $(N=14)$, that received both the ASC subcapsular injection and the oral treatment with Losartan. Sham-operated animals $(\mathrm{N}=11)$ were used as control.

Supplementary Figure 3: In vitro Experiments. (A) Illustrative microphotographs of immunofluorescence of cultured ASC, previously treated with: Normal rat serum (NRS), uremic 
rat serum (URS) or URS+10 $\mu \mathrm{M}$ of Losartan (URS+LOS). Cells positive for PCNA are stained in red. The total number of $\mathrm{ASC} / \mathrm{mm}^{2}$ in each experimental treatment is represented in the bar graph in B, while the number of PCNA+ASC $/ \mathrm{mm}^{2}$ is represented in C.

Supplementary Table 1: Genes and primer sequences. The housekeeping beta-actin (Bact) gene was used as an endogenous control of the PCR reaction.

Supplementary Table 2: Final parameters analyzed at the end of the study period: Survival rate (SR; \%), Body weight (BW; g), Urinary volume (UV; $\mathrm{mL})$, Serum creatinine concentration (Scr; $\mathrm{mg} / \mathrm{dL})$, Urinary creatinine concentration $(\mathrm{Ucr} ; \mathrm{mg} / \mathrm{dL})$, Creatinine clearance $(\mathrm{CrCl}$; $\mathrm{mg} / \mathrm{min} / \mathrm{RBSA}$ ), Blood urea nitrogen (BUN; mg/dL) and Renal hypertrophy. ${ }^{*}: p<0.05$ vs. Sham 30d; $§: p<0.05$ vs. Basal CKD, \#: $p<0.05$ vs. CKD; $+: p<0.05$ vs. CKD+ASC; $\neq: p<0.05$ vs. CKD+LOS. 


\section{DISCUSSION}

CKD is still a major global health issue. According to recent surveys, by the end of 2020 there were at least 2.5 million of patients receiving renal replacement therapy worldwide, and this number is expected to double by 2030. The high mortality rates and the highly debilitating character of CKD, together with the current lack of an effective treatment to halt its progression, urge the world to seek for new therapeutic strategies to detain the advance of CKD [22,23]. In the present study we sought to verify the potential renoprotective effects of cell therapy, using a high number of ASC $\left(2 \times 10^{6}\right)$, administered directly in the renal subcapsular area, in Wistar rats submitted to the $5 / 6$ renal ablation model of CKD. In addition, we aimed to investigate the effects of the association of this innovative therapeutic strategy with the pharmacological blockade of RAAS, currently employed in the conservative management of CKD.

As widely described in the literature, the $5 / 6$ renal ablation is a very consistent experimental model to mimic human progressive nephropathy $[9,24,25]$. Accordingly, in the present study, the animals submitted to this procedure exhibited severe arterial hypertension, loss of selectivity of the glomerular filtration barrier, evidenced by massive proteinuria and albuminuria, along with histological alterations of the renal parenchyma, such as glomerulosclerosis and tubulointerstitial inflammation and fibrosis, as early as 15 days after CKD induction. Similar to what is usually observed in human chronic nephropathy, these parameters worsened over time in untreated CKD animals, reaching exuberant values, and leading to elevated mortality rates after 30 days of renal ablation. Since most of the characteristic features of CKD were completely established 15 days after $5 / 6$ ablation, we choose this point to start the experimental treatments, to better investigate the potential effects achieved by these therapies on patients already affected by CKD. 
Our research group choose the renal subcapsular space to inoculate ASC, based on the traditional results obtained by Melgren and collaborators, using pancreatic islets. These authors demonstrated that the renal subcapsular space of diabetic mice offered better conditions for the growth of transplanted pancreatic islets, when compared to other sites of inoculation, such as the liver and spleen [26]. More recently, in an experimental study with rats, Svensson et. al also showed that transplanted pancreatic islets inserted in the renal subcapsular space presented greater revascularization and oxygenation, when compared to islets inoculated directly in the liver of these animals [27]. Corroborating the results obtained by Cavaglieri and collaborators, in an elegant study employing the 5/6 renal ablation model of CKD, our analysis of cell migration demonstrated the effective displacement and distribution of cells through the renal cortex of the animals that received the subcapsular renal inoculation of ASC, which were still present in the renal parenchyma until the end of the study, 30 days after renal ablation, thus consolidating the renal subcapsular pathway as a viable alternative for cell therapy, at least in experimental studies [10].

Here, we demonstrated that ASC alone slowed down the progression of proteinuria, albuminuria and glomerulosclerosis in animals that already exhibited significant renal injury. Furthermore, it partially detained ZO1 depletion observed in animals with CKD, thus preserving the tight junctions between podocyte processes and reducing the loss of macromolecules to the space of Bowman, suggesting a specific protective effect of ASC on the glomeruli. Our findings are in agreement with those of Ornellas et al., which demonstrated BMSC to protect rats submitted to a model of puromycin-induced glomerular injury from podocyte loss, from the effacement of the podocyte processes and from the loss of constitutive glomerular filtration barrier compounds such as nephrin, podocin, synaptopodin and podocalyxin [12]. Additionally, we showed that a single administration of ASC at 15 days of CKD was efficient in 
detaining the progression of established tubulointerstitial fibrosis, keeping the percentage of interstitial area of CKD+ASC animals at 30 days of renal ablation numerically similar to that observed before ASC injection. Akan and collaborators have recently shown similar results with the intravenous injection of human amnion-derived mSC (hAMSC) in this same CKD model. The authors observed a reduction in the interstitial expression of collagen and TGF- $\beta 1$ in the renal parenchyma of animals submitted to the cell therapy, accompanied by the increased expression of Bone Morphogenetic Protein-7 (BMP7), a member of the TGF- $\beta$ superfamily, which counteracts the biological functions of TGF- $\beta 1$, thus exhibiting anti-fibrotic properties $[28,29]$. Therefore, Tang et al. demonstrated that BMSC inoculation promoted a decrease in the renal expression of $\alpha$-SMA, collagen types I, II, III and TGF- $\beta 1$, in animals submitted to the adenine-overload model of interstitial fibrosis [30].

The development of RAAS blockers, more than 2 decades ago, was a major breakthrough for the management of CKD until the present days. Pharmacological RAAS blockade reduced the mortality of CKD patients by cardiovascular events, and considerably improved life quality, mainly due to its antihypertensive and antiproteinuric effects. Nevertheless, the reduction in proteinuria obtained with ACEls or ARBs is insufficient for a number of patients, moreover, these treatments delay but do not prevent CKD progression and renal function deterioration $[31,32]$. Corroborating what has been observed in human nephropathy, and in accordance with previous studies [24], here we demonstrated that oral treatment with $50 \mathrm{mg} / \mathrm{Kg} / \mathrm{d}$ of Losartan between the $15^{\text {th }}$ and the $30^{\text {th }}$ days completely prevented the mortality of rats submitted to the $5 / 6$ renal ablation model. Moreover, RAAS blockade in monotherapy reversed hypertension and slowed down but did not stop the progression of proteinuria and albuminuria. 
A number of in vitro studies have demonstrated All to stimulate the proliferation and activation of cultured fibroblasts [33]. Additionally, All can be described as a growth factor known to play a crucial role in epithelial-to-mesenchymal transdifferentiation in the development of tumor metastasis development, as well as in renal, alveolar and peritoneal epithelial cells [34]. In accordance with the publications employing cultured cells, experimental studies with different CKD models endorse the profibrotic and proinflammatory biological activities of All, which is described to act as a cytokine, positively regulating renal and immunological cell response of the beneficial effects of RAAS blockade on renal inflammation and fibrosis [33-36]. Accordingly, here we demonstrated that CKD+LOS animals exhibited significantly less renal interstitial fibrosis, and cell proliferation, as well as a slight reduction in both macrophage and lymphocyte infiltration in the renal parenchyma, compared to the untreated CKD rats.

Since the currently employed RAAS blockade is not enough to stop CKD progression, here we associated cell therapy to this oral treatment in order to verify if additional renoprotection would be achieved. This was the first study to associate the injection of ASC with a clinically employed pharmacological approach of RAAS blockade. We chose the ARB Losartan, instead of an ACEI, based on our in vitro findings, which showed that cultured ASC, stimulated with uremic rat serum, in order to better mimic the microenvironment of renal subcapsular space of CKD animals, presented greater proliferative capacity when treated with LOS diluted in the culture media, compared to cells cultivated in the absence of this drug, thus proving evidence that RAAS blockage did not impair ASC growth and survival, and even exerted stimulatory effects on these cells [20].

When associated to Losartan treatment, a single subcapsular inoculation of $2 \times 10^{6}$ ASC promoted a significant improvement in several parameters associated with the progression of 
CKD in the 5/6 renal ablation model; especially proteinuria and albuminuria, whose values did not only stop progressing, but also regressed with the combined therapy. Surprisingly, ASC+LOS association virtually normalized UPE and UAE in the 5/6 nephrectomy CKD model, since the $C K D+A S C+L O S$ group did not differ from the Sham group in these parameters. Moreover, the association of ASC to LOS promoted the regression of structural glomerular damage by reversing glomerulosclerosis and preserving ZO1 glomerular expression in the CKD animals more efficiently than the monotherapies. We also observed that ASC+LOS significantly detained hypertension and renal interstitial macrophage infiltration more effectively than ASC or LOS alone, also normalizing the renal expression of IL-6, a potent pro inflammatory mediator. 


\section{CONCLUSION}

In the present study, we demonstrated for the first time that the association of a subcapsular injection of ASC with RAAS blockade promoted greater renoprotection when compared to both strategies in monotherapy, thus suggesting cellular therapy with ASC to be a potential adjuvant to the current pharmacological approach used for CKD conservative management, although more studies are still required before the clinical use of ASC can be established. 


\section{DATA AVAILABILITY}

Full rough data supporting the findings of this study are available from the corresponding author upon request.

\section{CONFLICTS OF INTEREST}

The authors declare they have no conflicts of interest.

\section{FUNDING STATEMENT}

Financial support for the development of the present research study was provided by the São Paulo Research Foundation (FAPESP \#2017/26216-0).

\section{ACKNOWLEDGMENTS}

We would like to thank our laboratory team, namely the colleagues: Andreza Aparecida Santos, Anne Carolina da Silva Constantino, Arizla Benicio Vieira de Menezes, Débora Cristina de Sousa, Kelly Ribeiro Cascimiro, Marcia Ribalta and Wellisson Farias Pereira, for their excellent technical support.

\section{PREPRINT}

In accordance with Hindawi politics, the authors are going to deposit the present manuscript in the preprint server medRxiv within the next few days. 


\section{REFERENCES}

1. Saran R, Robinson B, Abbot KC, et al. US Renal Data System 2016 Annual Data Report: Epidemiology of Kidney Disease in the United States. Am J Kidney Dis. 69(3,1): A7-A8, 2017. doi: https://doi.org/10.1053/j.ajkd.2016.12.004.

2. Ruiz-Ortega M, Rayego-Mateos S, Lamas S, Ortiz A, Rodrigues-Diez RR. Targeting the progression of chronic kidney disease. Nat Rev Nephrol. 2020 May;16(5):269-288. doi: 10.1038/s41581-019-0248-y. Epub 2020 Feb 14. PMID: 3206048.

3. Butt, L., Unnersjö-Jess, D., Höhne, M. et al. A molecular mechanism explaining albuminuria in kidney disease. Nat Metab 2, 461-474. 2020. https://doi.org/10.1038/s42255-020-0204.

4. Noronha IL, Fujihara CK, Zatz R. The inflammatory component in progressive renal disease - are interventions possible? Nephrol Dial Transplant 17:363-368; 2002. https://doi.org/10.1093/ndt/17.3.363.

5. Fanelli C, Noreddin A, Nunes A.: Inflammation in nonimmune-mediated chronic kidney disease. In: Chronic Kidney Disease - From Pathology to Clinical Improvements, edited by Rath T, London, IntechOpen, 2018, pp 153-177. doi: 10.5772/intechopen.70611.

6. Rüster C, Wolf G. Renin-Angiotensin-Aldosterone System and Progression of Renal Disease. J Am Soc Nephrol. 2006; 17:2985-2991.doi: https://doi.org/10.1681/ASN.2006040356.

7. Fogo $A B$, et al. Renal Fibrosis: not Just PAI-1 in the Sky. J Clin Invest 112:326-328, 2003. doi: $10.1172 / \mathrm{JCl} 19375$.

8. Rodríguez-Iturbe B, García García G. The role of tubulointerstitial inflammation in the progression of chronic renal failure. Nephron Clin Pract. 2010;116(2):c81-8. doi: 10.1159/000314656. Epub 2010 May 22. PMID: 20502043.

9. Fanelli C, Arias SCA, Machado FG, et al. Innate And Adaptive Immunity are Progressively Activated in Parallel with Renal Injury in the 5/6 Renal Ablation Model. Sci Rep. Jun 9;7(1):3192, 2017. doi: 10.1038/s41598-017-02915-6.

10. Cavaglieri RC, Martini D, Sogayar MC, Noronha IL. Mesenchymal stem cells delivered at the subcapsule of the kidney ameliorate renal disease in the rat remnant kidney model. Transplant Proc. Apr;41(3):947-51, 2009. doi: 10.1016/j.transproceed.2009.01.072.

11. Noronha IL, Cavaglieri RC, Janz FL, et al. Zugaib M, Bydlowski SP. The potential use of stem cells derived from human amniotic fluid in renal diseases. Kidney Int Suppl Sep;1(3):7782, 2011. doi: 10.1038/kisup.2011.18.

12. Ornellas, F.M., Ramalho, R.J., Fanelli, C. et al. Mesenchymal Stromal Cells Induce Podocyte Protection in the Puromycin Injury Model. Sci Rep 9, 19604 (2019). https://doi.org/10.1038/s41598-019-55284-7. 
13. Bassi EJ, Aita CAM, Camara NOS. Immune regulatory properties of multipotent mesenchymal stromal cells: Where do we stand? World J Stem Cells. 3(1): 1-8, 2011. doi: 10.4252/wjsc. v3.i1.1.

14. Liu J, Liu Q, Chen X. The Immunomodulatory Effects of Mesenchymal Stem Cells on Regulatory B Cells. Front Immunol. 2020 Aug 14;11:1843. doi: 10.3389/fimmu.2020.01843. PMID: 32922398; PMCID: PMC7456948.

15. Xing $L$, Song $E, Y u C Y$, et al. Bone marrow-derived mesenchymal stem cells attenuate tubulointerstitial injury through multiple mechanisms in UUO model. J Cell Biochem. 2019 Jun;120(6):9737-9746. doi: 10.1002/jcb.28254. Epub 2018 Dec 7. PMID: 30525227.

16. Pepineli R, Gouveia PQ, Garnica MR, Fanelli C, et al. (2020) Mesenchymal Stem Cells Derived from Adipose Tissue Ameliorate Chronic Allograft Rejection in the Long Term in a Rat Experimental Model of Kidney Transplantation. J Stem Cell Res Dev Ther 6: 051. doi:10.24966/SRDT-2060/100051.

17. Costalonga EC, Fanelli C, Garnica MR, Noronha IL. Adipose-Derived Mesenchymal Stem Cells Modulate Fibrosis and Inflammation in the Peritoneal Fibrosis Model Developed in Uremic Rats. Stem Cells Int. 2020 May 20;2020:3768718. doi: 10.1155/2020/3768718. PMID: 32565826; PMCID: PMC7256710.

18. Mancini G, Carbonara AO, Heremans JF: Immunochemical quantification of antigens by single radial immunodiffusion. Immunochemistry 2:235-254, 1965. doi: 10.1016/00192791(65)90004-2.

19. Jepsen FL, Mortensen PB. Interstitial fibrosis of the renal cortex in minimal change lesion and its correlation with renal function: a quantitative study. Virchows Arch A Pathol Anat Histol 383:265-70, 1979. doi: 10.1007/BF00430245.

20. Jang HR, Cho HJ, Zhou Y, Shao NY, et al. Modeling Uremic Vasculopathy With Induced Pluripotent Stem Cell-Derived Endothelial Cells as a Drug Screening System. Front Cell Dev Biol. 2021 Jan 12;8:618796. doi: 10.3389/fcell.2020.618796. PMID: 33511129; PMCID: PMC7835337.

21. Wallenstein S, Zucker CL, Fleiss JL: Some statistical methods useful in circulations research. Circ Res 47:1-9, 1980. doi: 10.1161/01.res.47.1.1.

22. GBD Chronic Kidney Disease Collaboration. Global, regional, and national burden of chronic kidney disease, 1990-2017: a systematic analysis for the Global Burden of Disease Study 2017. Lancet. 2020 Feb 29;395(10225):709-733. doi: 10.1016/S01406736(20)30045-3. Epub 2020 Feb 13. PMID: 32061315; PMCID: PMC7049905.

23. Wilson S, Mone P, Jankauskas SS, et al. Chronic kidney disease: Definition, updated epidemiology, staging, and mechanisms of increased cardiovascular risk. J Clin Hypertens (Greenwich). 2021 Apr;23(4):831-834. doi: 10.1111/jch.14186. Epub 2021 Jan 17. PMID: 33455061; PMCID: PMC8035205.

24. Arias SCA, Valente CP, Machado FG, Fanelli C, et al. Regression of Albuminuria and Hypertension and Arrest of Severe Renal Injury by a Losartan-Hydrochlorothiazide 
Association in a Model of Very Advanced Nephropathy. PLoS ONE 8(2): e56215. doi: 10.1371/journal.pone.0056215, 2013.

25. Yang HC, Zuo Y, Fogo AB. Models of chronic kidney disease. Drug Discovery Today: Disease Models. Volume 7. Issues 1-2, 2010. Pages 13-19, ISSN 1740-6757. https://doi.org/10.1016/j.ddmod.2010.08.002.

26. Mellgren A, Schnell Landström AH, Petersson B, et al. The renal subcapsular site offers better growth conditions for transplanted mouse pancreatic islet cells than the liver or spleen. Diabetologia. 1986 Sep;29(9):670-2. doi: 10.1007/BF00869269. PMID: 3098614.

27. Svensson J, Lau J, Sandberg M, et al. High vascular density and oxygenation of pancreatic islets transplanted in clusters into striated muscle. Cell Transplant. 2011;20(5):783-8. doi: 10.3727/096368910X536527. Epub 2010 Nov 5. PMID: 21054943.

28. Akan E, Cetinkaya B, Kipmen-Korgun D, Ozmen A, et al. Effects of amnion derived mesenchymal stem cells on fibrosis in a 5/6 nephrectomy model in rats. Biotech Histochem. 2021 Feb 1:1-14. doi: 10.1080/10520295.2021.1875502. Epub ahead of print. PMID: 33522283.

29. Silva FMO, Costalonga EC, Silva C, et al. Tamoxifen and bone morphogenic protein-7 modulate fibrosis and inflammation in the peritoneal fibrosis model developed in uremic rats. Mol Med. 2019;25(1):41. Published 2019 Aug 28. doi:10.1186/s10020-019-0110-5.

30. Tang, H., Zhang, P., Zeng, L. et al. Mesenchymal stem cells ameliorate renal fibrosis by galectin-3/Akt/GSK3ß/Snail signaling pathway in adenine-induced nephropathy rat. Stem Cell Res Ther 12, 409. 2021. https://doi.org/10.1186/s13287-021-02429-z.

31. Romero CA, Orias M, Weir MR. Novel RAAS agonists and antagonists: clinical applications and controversies. Nat Rev Endocrinol. 2015 Apr;11(4):242-52. doi: 10.1038/nrendo.2015.6. Epub 2015 Feb 10. PMID: 25666495; PMCID: PMC7097622.

32. Lambers Heerspink HJ, de Borst MH, Bakker SJ, Navis GJ. Improving the efficacy of RAAS blockade in patients with chronic kidney disease. Nat Rev Nephrol. 2013 Feb;9(2):11221. doi: 10.1038/nrneph.2012.281. Epub 2012 Dec 18. PMID: 23247573.

33. Burns WC, Velkoska E, Dean R, Burrell LM, et al. Angiotensin II mediates epithelial-tomesenchymal transformation in tubular cells by ANG 1-7/MAS-1-dependent pathways. Am J Physiol Renal Physiol. 2010 Sep;299(3):F585-93. doi: 10.1152/ajprenal.00538.2009. Epub 2010 Jun 16. PMID: 20554647.

34. Ruiz-Ortega $M$, Rupérez $M$, Esteban $V$, et al. Angiotensin II: a key factor in the inflammatory and fibrotic response in kidney diseases. Nephrol Dial Transplant. 2006 Jan;21(1):16-20. doi: 10.1093/ndt/gfi265. Epub 2005 Nov 9. PMID: 16280370.

35. Sanz AB, Ramos AM, Soler MJ, et al. Advances in understanding the role of angiotensinregulated proteins in kidney diseases. Expert Rev Proteomics. 2019 Jan;16(1):77-92. doi: 10.1080/14789450.2018.1545577. Epub 2018 Nov 27. PMID: 30412432.

36. Andersen $S$, Mischak $H$, Zürbig $P$, et al. Urinary proteome analysis enables assessment of renoprotective treatment in type 2 diabetic patients with microalbuminuria. BMC 
bioRxiv preprint doi: https://doi.org/10.1101/2021.09.20.461095; this version posted September 20, 2021. The copyright holder for this preprint (which was not certified by peer review) is the author/funder. All rights reserved. No reuse allowed without permission.

Nephrol. 2010 Nov 1;11:29. doi: 10.1186/1471-2369-11-29. PMID: 21040538; PMCID: PMC2988775. 